\title{
le bilan énergétique en mécanique des roches
}

Ch. Fairhurst

Professeur \& Président de Section Department of Civil \& Mineral Engineering University of Minnesota Minneapolis, Minnesota U.S.A. 


\section{LE BILAN ENERGETIQUE EN MECANIQUE DES ROCHES}

L'article étend à la mécanique des roches une conception générale basée sur le bilan énergétique des phénomènes. Cette approche est appliquée aux problèmes suivants qu'elle éclaire d'un jour nouveau :

- fracturation hydraulique et abattage à l'explosif:

- stabilité de la roche en compression ;

- stabilité des tunnels ;

- stabilité d'un massif formé d'un assemblage de blocs :

- dimensionnement des piliers de mine.

Les nouvelles possibilités du traitement conversationnel sur écran cathodique sont largement exploitées.

\section{THE APPLICATION OF MECHANICS TO ROCK ENGINEERING}

The article extends the general energy balance concept to rock mechanics. This approach is used to throw new light on:

- hydraulic fracturing and rock blasting:

- stability of rock in compression;

- tunnel stability;

- stability of block-jointed rock;

- mine pillar design.

The new possibilities of computer interactive graphics are discussed. 


\title{
le bilan énergétique en mécanique des roches
}

\author{
par Ch. FAIRHURST
}

\begin{abstract}
"...Une troisième impression est l'absence comparable d'une base théorique dans la plupart des réalisations de l'ingénieur et ceci jusqu'il y a une centaine d'années. Cela ne veut pas dire pour autant que le climat intellectuel créé par Newton, Hooke, Boyle et d'autres durant la révolution scientifique ne préparait pas les fondations du bouleversement industriel qui s'en suivit; mais il ne semble pas que les ingénieurs eux-mêmes en aient été conscients. Pendant plus d'un siècle, ils se passèrent de toute théorie et lorsque la science commença à toucher la technique ces ingénieurs - ou plutôt leurs successeurs - prirent lentement conscience que les temps avaient changé et que les progrès futurs allaient devoir se faire avec la physique et la chimie. Peut-on en tirer une leçon valable pour nous aujourd'hui? »
\end{abstract}

\author{
Extrait de "Can We Learn from History ". \\ Introduction de "Engineering Heritage ". \\ Institut des Ingénieurs mécaniciens \\ (Londres) 1963, p. 180.
}

\section{INTRODUCTION}

Le seul fait de constater dans la nature la complexité des structures géologiques nous fait comprendre pourquoi le développement de bases théoriques rationnelles, applicables au dimensionnement des ouvrages au rocher, a été lent à côté des progrès réalisés dans le dimensionnement des structures utilisant uniquement des matériaux fabriqués par l'homme. Les premières tentatives d'utilisation pour les roches des concepts et des procédés mis au point pour les matériaux fabriqués par l'homme n'eurent en général qu'un succès limité, à cause de la simplification exagérée des données géologiques et à cause d'hypothèses acceptées sans étude critique. Le dimensionnement des ouvrages au rocher reste un " art plutôt qu'une science " et les seules règles existantes sont empiriques.

Il va sans dire que cette situation doit changer. Les travaux au rocher deviennent de jour en jour plus ambitieux et l'extrapolation à partir d'expériences antérieures ne cesse de s'amplifier. Les ouvrages souterrains sont édifiés dans des sites moins favorables. De nouvelles applications telles que l'exploitation de l'énergie géothermique, le rejet de déchets nucléaires, le stockage de l'énergie hydro-électrique, l'utilisation de l'espace souterrain pour une multitude d'usages, nécessitent toutes une compréhension plus fine du comportement des massifs rocheux. Comme ce fut le cas pour nos prédécesseurs, les ingénieurs-mécaniciens de la fin du $\mathrm{XIX}^{\mathrm{e}}$ siècle, nous commençons à ressentir la nécessité de meilleurs fondements théoriques de la mécanique des roches appliquée.

En établissant ces fondements, nous devons être conscients que nous travaillons avec un tout autre matériau que celui des ingénieurs-mécaniciens. Le matériau géologique - la roche - possède des caractéristiques variables, jusqu'à un certain degré imprévisibles, que nous négligeons à nos risques et périls. Des principes généraux fondamentaux doivent être énoncés afin de servir de guide lors du dimensionnement des ouvrages, pourvu qu'ils soient utilisés avec intelligence en fonction des données naturelles et par des hommes d'expérience. Labasse [20] disait déjà cela au sujet du dimensionnement du soutènement des tunnels dans la roche (voir page 32).

La mise en œuvre de ces démarches, basées sur une théorie saine, a été le but principal de la recherche en mécanique des roches effectuée à l'Université du Minnesota durant les vingt dernières années. La présente communication décrit quelques-uns de ces développements et donne des exemples de recherche provenant de l'Université ou d'ailleurs, qui concernent des problèmes de grande importance pratique. Dans chaque cas particulier, les détails des calculs pourront être trouvés dans les ouvrages cités en référence.

\section{RESISTANCE ET STABILITE}

L'objectif final du projeteur est d'obtenir la certitude que la structure ne se rompra pas en service. Dans la plupart des cas, "rupture " est synonyme de limite élastique ou de "contrainte limite ". Mais dans certaines situations, le dépassement local de cette contrain- te limite ou résistance fait que les forces appliquées sont redistribuées dans la structure et il est fort possible qu'au total cette dernière puisse toujours jouer le rôle pour lequel elle a été conçue, c'est-à-dire qu'il n'y a pas réellement " rupture ». 
Très souvent, les ouvrages au rocher font partie de cette seconde catégorie. La résistance de la roche est généralement inconnue et peut varier de façon spectaculaire d'un point à un autre. Au surplus, les charges appliquées sont pareillement inconnues. Considérant ces caractéristiques, il est en général plus réaliste d'analyser les problèmes concernant la roche en termes de "stabilité " structurelle plutôt que de considérer sa résistance; en d'autres mots, il est préférable de se poser la question suivante : " Qu'arriverait-il si la résistance (inconnue) était dépassée ?».

\section{THEORIE DE LA RUPTURE D'APRES GRIFFITH}

L'exemple le plus courant d'analyse de la stabilité en mécanique des roches est probablement la théorie de la rupture selon Griffith, dont certains détails sont revus dans l'annexe à cette communication.

Dans son principe, cette théorie est une application du principe de l'énergie potentielle minimale. Elle établit qu'après initiation, une fissure de traction se propagera dans un matériau fragile lorsque l'énergie libérée par la fracturation $\left(\mathrm{E}_{s}\right)$ dépasse l'énergie nécessaire pour créer de nouvelles surfaces de fractures $\left(\mathrm{E}_{d}\right)$.

$$
\frac{\partial \mathrm{E}_{s}}{\partial c} \geq \frac{\partial \mathrm{E}_{d}}{\partial c}
$$

Dans cette relation $c$ représente la longueur de la fissure.

Développée par Griffith afin d'expliquer la résistance des matériaux fragiles, la théorie de la rupture est à la base de la Mécanique de la Fracturation [3] et fut utilisée avec succès pour interpréter le comportement des matériaux à la rupture. L'application de cette théorie à l'analyse des fissures de traction dans les roches aboutit à des résultats très intéressants dont certains sont décrits maintenant.

\section{ESSAIS DE TRACTION: INDIRECTE SUR LES ROCHES}

La difficulté inhérente à l'essai de traction directe a conduit au développement d'un certain nombre d'essais indirects, dans lesquels la traction est induite dans une partie seulement de l'échantillon, par application d'un chargement local en compression. La théorie de l'élasticité est ensuite utilisée afin d'interpréter les résultats, en reliant la charge de rupture à la contrainte maximale de traction obtenue dans l'échantillon. L'hypothèse usuelle est que la rupture se produit à partir de la contrainte maximale de traction. Il est bien connu que la « résistance à la traction " déterminée de cette façon varie largement d'un type d'essai à l'autre pour la même roche.

Hardy [4] a utilisé les notions de bilan énergétique de Griffith pour interpréter les résultats de trois types d'essais de traction indirecte, sur deux types de granite : a) l'essai de poutre (avec ou sans entaille), b) l'essai dit "Brésilien ", c) l'essai d'anneau. La variation théorique de l'énergie du système de chargement (poutre ou disque) a été calculée en fonction de la longueur de la fissure en utilisant la méthode des éléments finis. La figure 1 décrit les différents essais ainsi que les "structures équivalentes " utilisées dans l'analyse numérique. Les tableaux 1 et 2 donnent les résultats obtenus pour le granite gris et le granite rouge (cristaux plus gros)

Le modèle théorique nécessite la connaissance des valeurs spécifiques de deux paramètres, " $\gamma$ » le travail de fracturation et « $c$ » la longueur initiale de la fissure. Les valeurs de " $\gamma$ " et " $c$ » ont été déterminées par ajustement des valeurs théoriques aux résultats obtenus pour les deux premiers essais donnés dans les tableaux 1 et 2 (les valeurs obtenues pour $\gamma$ et $c$ sont mentionnées en haut de la troisième colonne). Ces valeurs furent ensuite utilisées pour calculer la force maximale de rupture $\left(\mathrm{F}_{p}\right)$ dans les autres essais. Bien que l'accord ne soit pas parfait, les résultats sont bien meilleurs que ceux qu'on obtient en utilisant la théorie de l'élasticité et ils sont remarquablement bons lorsque l'on considère la complexité de la structure rocheuse par rapport au modèle analysé qui ne contient qu'une seule fissure. Ces résultats démontrent clairement les insuffisances du critère de la contrainte de traction maximale dans le cas des essais de traction indirecte sur échantillons de roche.

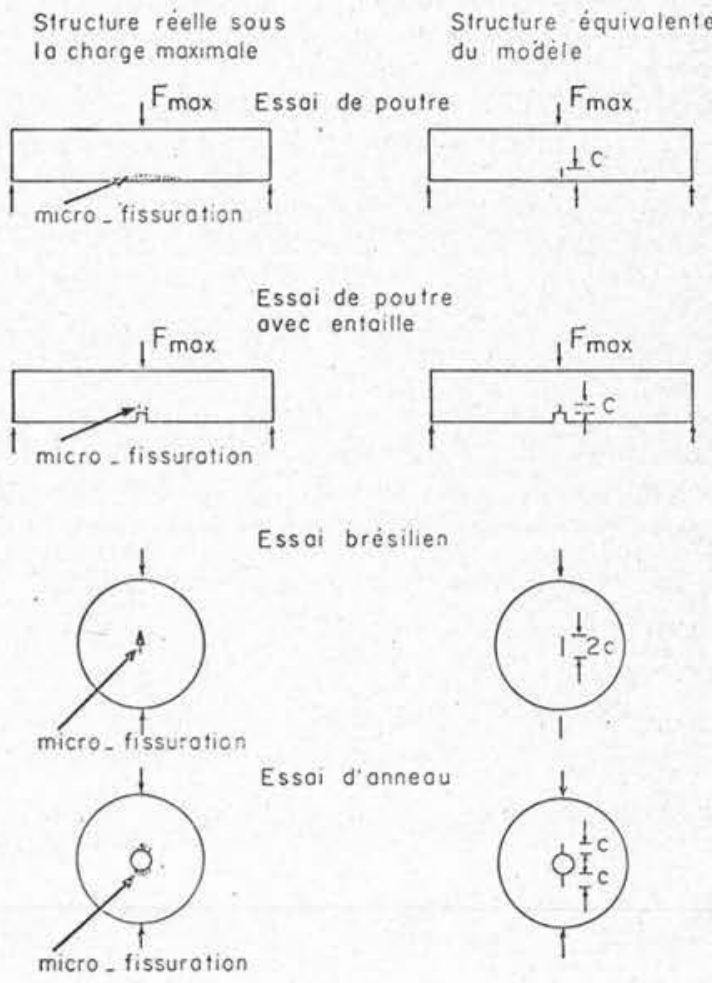

Fig. 1. - Structures équivalentes utilisées dans le modèle de fracturation de la roche. 
TABLEAU 1. - Résumé des résultats - Granit gris.

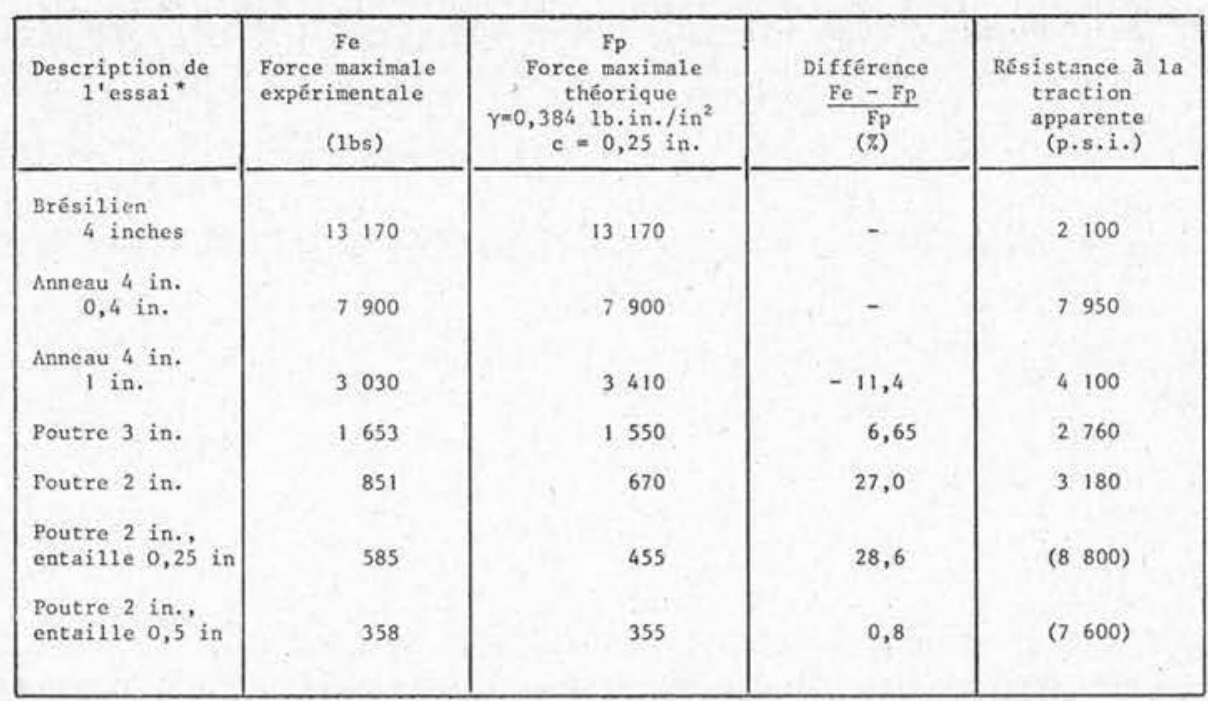

* N.B. Epaisseur dés échantillons 1 in. ; portếe de la poutre 10 in.

TABLEAU 2, - Résumé des résultats - Granit rouge.

\begin{tabular}{|c|c|c|c|c|}
\hline $\begin{array}{c}\text { Description de } \\
\text { I'essai }\end{array}$ & $\begin{array}{c}\text { Fe } \\
\text { Force maximale } \\
\text { expérimentale } \\
\text { (1bs) }\end{array}$ & 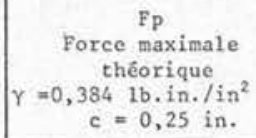 & $\begin{array}{l}\text { Différence } \\
\frac{\text { Fe - Fp }}{F_{p}} \\
(\bar{z})\end{array}$ & $\begin{array}{c}\text { Résistance à la } \\
\text { traction } \\
\text { apparente } \\
\text { (p.s.i.) }\end{array}$ \\
\hline Poutre 2 in. & 678 & 670 & 1,2 & 2540 \\
\hline $\begin{array}{l}\text { Poutre } 2 \text { in., } \\
\text { entaille } 0,25 \text { in }\end{array}$ & 466 & 455 & 2,4 & $(7000)$ \\
\hline $\begin{array}{l}\text { Poutre } 2 \text { in., } \\
\text { entaille } 0,50 \text { in }\end{array}$ & 356 & 355 & 3,0 & (7 500$)$ \\
\hline Poutre 3 in. & 955 & 1550 & 31,2 & 1605 \\
\hline $\begin{array}{l}\text { Poutre } 3 \text { in., } \\
\text { entaille } 0,50 \text { in }\end{array}$ & 750 & 864 & 14,7 & $(7060)$ \\
\hline
\end{tabular}

* N.B. Epaisseur des échantillons $1 \mathrm{in}$. ; portée de la poutre 10 in.

\section{FRACTURATION HYDRAULIQUE ET ABATTAGE A L'EXPLOSIF}

Dans une autre application de la méthode énergétique, Hardy a examiné la question de la propagation des fissures dans la fracturation hydraulique.

La fracturation hydraulique est une technique utilisée pour augmenter la production des champs pétrolifères. Une section du puits est mise en pression jusqu'au moment où une fracture est initiée à la paroi et se propage dans le massif rocheux, créant une zone de grande perméabilité qui facilite l'écoulement du pétrole vers le puits. En adaptant cette technique à la mesure des contraintes in situ, la question se pose de la détermination de la variation de la pression dans le forage en fonction de la longueur de la fissure $(a)$ au cas où le fluide pénètre la fissure et $(b)$ au cas où le fluide est maintenu dans le forage au moyen d'une membrane imperméable. Les résultats de cette analyse sont donnés figure 2.

Des résultats similaires ont été obtenus indépendamment par Ouchterlony [5] dans le cas de propagation de fractures au moyen d'explosifs.

Fig. 2. - Pression en fonction de la longueur de la fissure dans le cas où cette dernière se propage à partir d'un forage.

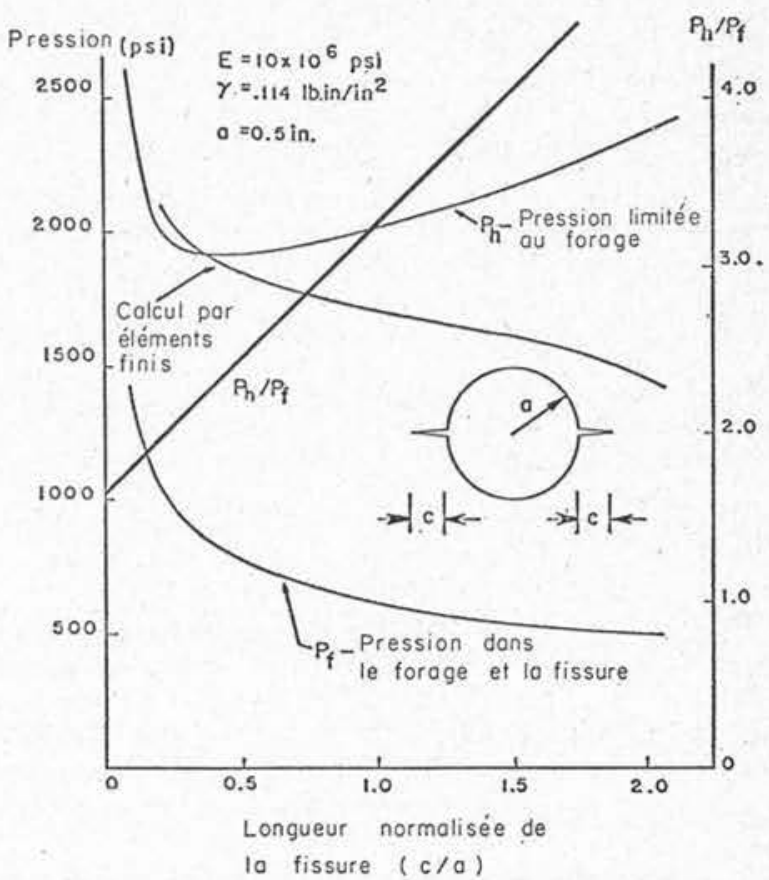


La conclusion majeure est que la pression nécessaire à la propagation de la fracture au fur et à mesure que celle-ci s'allonge devient rapidement beaucoup plus grande dans le cas où le fluide (gaz ou liquide) reste dans le forage que dans le cas où le fluide peut pénétrer dans la fissure.

Ce résultat a des implications importantes dans le cas de l'abattage à l'explosif. Bligh [6] a analysé la mise en pression dynamique de la roche. Il affirme que la montée très rapide de la pression de l'explosif fait que la contrainte tangentielle à la paroi du forage est instantanément une compression. On comprend pourquoi cette combinaison produit une pulvérisation intense à proximité de la paroi du forage, les fissures radiales ne se développant qu'à une certaine distance (petite) de la paroi. Les gaz à haute pression sont confinés dans le forage par cet anneau de roche pulvérisée et ne participent donc pas activement à la propagation des fissures. Cela suggère que les explosifs industriels traditionnels provoquent l'extension des fractures suivant la courbe $\mathrm{P}_{h}$ de la figure 2 , c'est-à-dire avec une pression nécessaire pour étendre les fissures qui augmente constamment. De cette façon une grande quantité d'énergie de pression est accumulée dans le forage lors de l'extension de la fracturation.

Ce mécanisme pourrait expliquer en partie le violent dégagement d'énergie associé à l'abattage à l'explosif ; lorsque les fissures atteignent la surface libre, l'énergie contenue dans le forage est libérée de façon soudaine dans un massif rocheux qui est déjà désorganisé. Bligh a fait des essais de tirs à pression (gazeuse) contrôlée afin de minimiser cette action d'étanchement par la roche pulvérisée et il affirme obtenir les meilleurs résultats avec des pressions faibles. Le fait d'utiliser de petites charges explosives dans des forages de grand diamètre afin d'obtenir un pré-découpage efficace est peut-être un indice supplémentaire du fait qu'une pression moindre, appliquée plus lentement, peut améliorer la propagation dynamique des fissures. Les promesses de cette technique pour améliorer l'abattage à l'explosif (moins violent, moins de vibrations, moins de poussière) engagent à entreprendre des études approfondies sur ce sujet.

Cornet [7] a examiné la question de la propagation des fissures dues aux explosifs à proximité d'une surface libre et a déterminé, au moyen de la méthode du bilan énergétique, quel était le chemin le plus probable suivi par les fissures engendrées par la mise en pression simultanée de deux forages.

La figure 3 est une coupe à travers deux forages cylindriques. Il est évidemment souhaitable que les fractures se rejoignent afin d'obtenir un tir efficace.

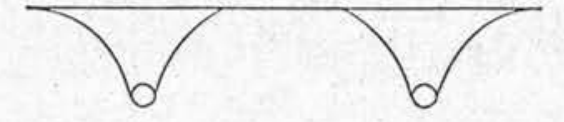

(a) Espocement trop grand

Formation de cratères individuels

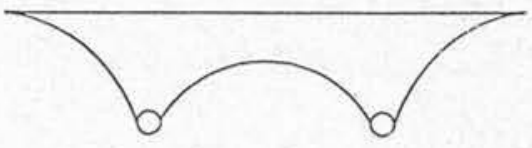

(b) Espacement proche de l'optimun Reloi entre fractures

Fig. 3. - Formation d'un cratère et variation du rapport couverture/espacement.

Les formules empiriques utilisées pour le tir dans les mines à ciel ouvert suggèrent que le quotient optimal espacement (S) sur couverture (B) devrait être de l'ordre de 1.5 à 2.0. Les études théoriques indiquent l'apparition d'une interconnection pour ces valeurs, d'autant meilleure qu'il existera des contraintes de compression parallèles à la surface libre ; la connection entre les trous de forages sera d'autant plus directe (en ligne droite) que les gaz pénètreront mieux dans la fissure. Ces résultats préliminaires suggèrent encore qu'une méthode d'analyse énergétique pourrait aboutir à une meilleure compréhension des facteurs gouvernant le tir et suggèrent également des moyens d'optimisation qui n'étaient pas apparents à partir des formules empiriques. Il est aussi encourageant de constater que les règles de la pratique courante confirment la validité probable du modèle de "propagation de la fracturation " dans la formation des cratères.

Il est important de noter que l'approche commune, utilisant la mécanique de la fracturation développée originellement par Irwin [8] et dans laquelle les coefficients d'intensité de contrainte sont calculés pour diverses configurations de fissuration plane, ne convient pas aux problèmes tels que celui qui vient d'être décrit et pour lesquels la fissure change d'orientation lors de sa propagation. Pour de tels problèmes, il est préférable de calculer directement les variations d'énergie en fonction d'un incrément de la longeur de la fissure dans différentes directions.

\section{ANALYSE DE LA STABILITE DE LA ROCHE EN COMPRESSION}

L'application de la théorie du bilan énergétique de Griffith au cas de la propagation de fractures de traction en est évidemment l'utilisation la plus directe. On peut toutefois appliquer les mêmes principes aux cas où la roche est en compression, comme cela a été fait par Cook [9] lors de son étude classique des coups de terrain dans les mines d'or d'Afrique du Sud.

Plus récemment, à l'Université du Minnesota, des techniques similaires ont été utilisées pour étudier le problème des coups de toit dans des mines de charbon américaines. Dans ce dernier cas, il fut nécessaire de tenir compte du comportement non linéaire du charbon.
Considérons la situation hypothétique de la figure 4, représentant une exploitation horizontale par chambres et piliers. Pour cette discussion nous supposerons que le pilier central est remplacé par un très grand vérin hydraulique. Le toit et le mur sont supposés se comporter de façon élastique. La charge de la couverture est distribuée entre les stots, les piliers et le vérin.

Dans la figure 4 , le chargement initial total transmis au vérin est désigné par $\mathrm{F}_{o}$. Au fur et à mesure que le vérin se contracte, la force diminue car la charge est transférée aux piliers et aux stots. Le toit s'affaisse et dans certains cas le mur se soulève. Tant que le toit reste 


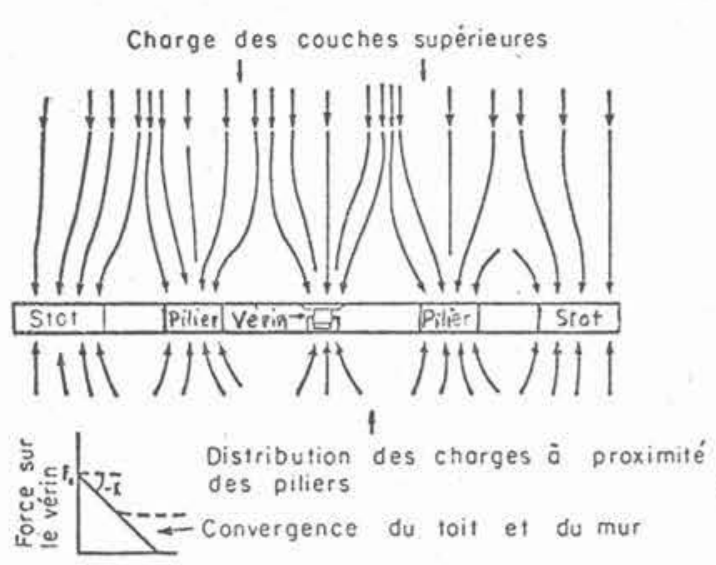

(a)

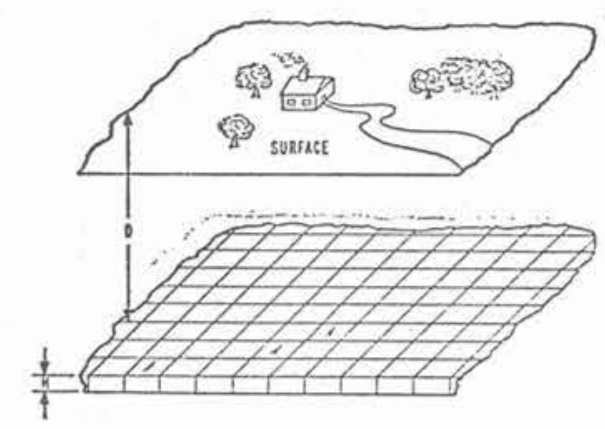

Division du filon en surfaces élémentoires

( b )

Fig. 4. - a) Distribution de la charge de la ccuverture au niveau de l'excavation horizontale. b) Subdivision du gisement en surfaces élémentaires.

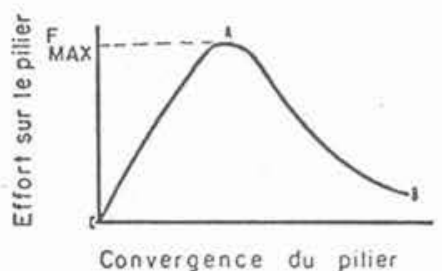

Courbe complete Effort - convergence J'un pilier de mine

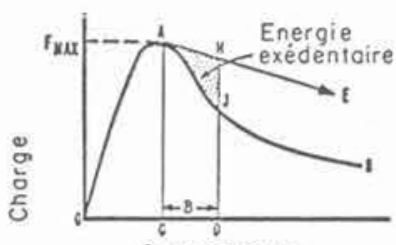

Convergence

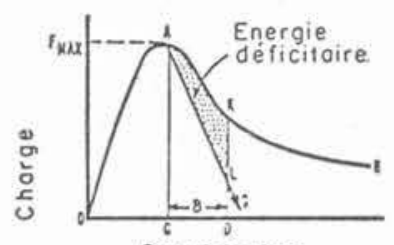

Convergence
Pilier Jans une zone de foible raideur INSTABLE
Pilier dans une zone

de forte roideur

STABLE.

(c)

Fig. 5. - Interaction entre les différentes « rigidités locales de la mine * et les courbes effort-déformation des éléments du gisement.

intact, la relation entre la force du vérin $(\mathrm{F})$ et la convergence $(\delta)$ ressemble à la ligne en trait continu de la figure $4 \mathrm{~A}$. Finalement, le vérin pourra être enlevé et la convergence finale du toit sera de $\tilde{\delta}_{o}$. Le travail effectué par le vérin peut être représenté par la surface triangulaire $\left(\mathrm{OF}_{o} \delta_{o}\right)$. Si le toit se fissure à un certain stade de la convergence, le vérin devra supporter la charge gravitationnelle (poids mort) de sorte que la courbe force/convergence suivra la courbe en pointillé indiquée figure $4 \mathrm{~A}$. La pente négative $"-\mathrm{K}$ » de la courbe dépendra des propriétés mécaniques du toit et du mur, de la largeur des chambres, de l'épaisseur des piliers adjacents et des stots, de la position du vérin dans la chambre, etc... Nous dirons que $\mathrm{K}$ est la " rigidité locale de la mine ".

Si nous remplaçons maintenant le vérin par un pilier, sa déformation - qui correspond à une contraction du vérin - dépendra de la force exercée par le toit et le mur. Un diagramme de la force en fonction de la déformation (convergence) est représenté figure $5 \mathrm{~A}$. Lorsque le pilier est chargé, il se déforme en suivant la ligne OA jusqu'à ce qu'il atteigne sa portance maximale $\left(\mathrm{F}_{\max }\right)$.

A ce moment là, une fracturation interne du pilier se produit, avec peut-être un certain écaillage des faces, de telle sorte que le pilier continue à se déformer sous charge réduite en suivant la courbe $\mathrm{AB}$. Le comportement du pilier au-delà du point $A$ dépendra de la force exercée par le toit, selon qu'elle sera suffisante ou non pour continuer la déformation. Ceci est illustré par les figures $5 \mathrm{~A}$ et $5 \mathrm{~B}$ dans lesquelles deux rigidités locales de la mine $\mathrm{AE}$ et $\mathrm{AG}$, ont été considérées et ont été superposées à la courbe chargement/déformation du pilier. Dans la figure $5 \mathrm{~B}$, une augmentation supposée
" $\delta$ " de la convergence - au delà du point $\mathrm{C}$ correspondant à $\mathrm{F}_{\max }$ - résulterait en une force $\mathrm{F}_{\mathrm{H}}$ exercée par le toit, alors que le pilier ne pourrait supporter qu'une valeur inférieure $F_{J}$. Cette situation est sans aucun doute instable et le pilier s'effondrera rapidement dès que $\mathrm{F}_{\max }$ sera atteint. La différence entre l'énergie générée par la mine (surface au-dessous de AE) et l'énergie absorbée par le pilier (surface au-dessous de la courbe AJB) différence représentée par une aire telle que $\mathrm{AHJ}$ lorsque la convergence atteint $\mathrm{D}$, est l'excès d'énergie disponible pour accélérer l'effondrement (devenu explosif) du pilier. Il est probable que la tangente à la courbe AJB dépendra de la vitesse de déformation, mais son comportement général est essentiellement inchangé.

Dans le second cas, représenté figure $5 \mathrm{C}$, la situation est inverse. Le toit est incapable de fournir la force nécessaire pour déformer le pilier au-delà du chargement maximal $\left(\mathrm{F}_{\max }\right)$ de sorte que la situation est stable ; une rupture contenue se produira, sans effondrement total. En pratique, la rigidité locale varie tout au long de la vie du pilier de sorte que le pilier peut avoir successivement les deux comportements, stable et instable, pour différentes valeurs de la convergence. Par exemple, une instabilité soudaine peut se produire après un tir.

Dans la discussion précédente, on a supposé que le pilier se comporterait de façon identique à ce qui est représenté figure $5 \mathrm{~A}$, correspondant au cas d'un pilier étroit, non soumis à une contrainte de confinement, c'est-à-dire libre de se déformer latéralement. Pour les parties du gisement éloignées des chambres, la compression verticale est associée à une résistance à l'expansion 
latérale et, par conséquent, la résistance au pic de ces parties est augmentée.

Pour les sections qui sont totalement empêchées de se déplacer latéralement, la charge maximale peut devenir très importante. La simulation de tel ' is conditions en laboratoire produit des courbes charge/déformation comme celles de la figure 6 . Notez que la rigidité élastique n'est pratiquement pas influencée par le confinement mais la charge maximale est augmentée de façon considérable.

Dans le modèle utilisé pour étudier le problème des coups de toits dans les mines de charbon le gisement est subdivisé en un nombre d'éléments carrés d'égales dimensions; l'une des caractéristiques charge/déformation $(1,2,3,4$ dans la figure 6$)$ est affectée à chacun des éléments; cette caractéristique est choisie en fonction de la proximité de la taille. Un élément peut toutefois changer de caractéristique, pour tenir compte des variations de confinement accompagnant l'approche de la taille. Le gisement est donc remplacé par un certain nombre de supports individuels, chacun d'entre-eux se déformant suivant l'une des quatre caractéristiques sous l'action de la gravité (notez que ce système peut également être utilisé pour des gisements verticaux - ou presque verticaux - en remplaçant la gravité par les contraintes horizontales in situ). Cette charge sera distribuée de manière élastique dans le toit et le mur d'après le comportement charge/déformation de chacun des supports individuels (éléments du gisement). par chaque fiche vers la terre est analogue à la force exercée par la gravité sur l'élément correspondant du gisement. Le voltage existant entre le noud et la terre lorsque la fiche est enlevée représente le déplacement (la convergence) qui apparaît lorsque cet élément est excavé.

Le produit : $\left(\frac{1}{2}\right)$. (courant avant enlèvement de la fiche) . (voltage après) représente l'énergie dissipée par le toit et le mur, au cours de l'excavation de cet élément du gisement.

Ce procédé par modèle analogique a été utilisé avec succès en Afrique du Sud [9] pour l'étude des diverses possibilités d'exploitation d'un gisement, en essayant de minimiser les libérations locales de hauts niveaux énergétiques, pouvant résulter en des coups de terrain dans les filons quartzitiques orifères.

L'utilisation de résistances constantes, correspondant à l'hypothèse d'un gisement élastique, conduit à des concentrations de contrainte toujours maximales au bord des excavations. Bien que cette hypothèse soit raisonnable dans le cas du quartzite d'Afrique du sud, elle n'est pas réaliste lorsque le gisement se comporte non linéairement, car alors les contraintes maximales (et les libérations d'énergie) sont distribuées d'une tout autre façon.

Le comportement non linéaire doit être introduit afin d'obtenir un accord convenable entre les prédictions faites d'après le modèle et l'expérience pratique des

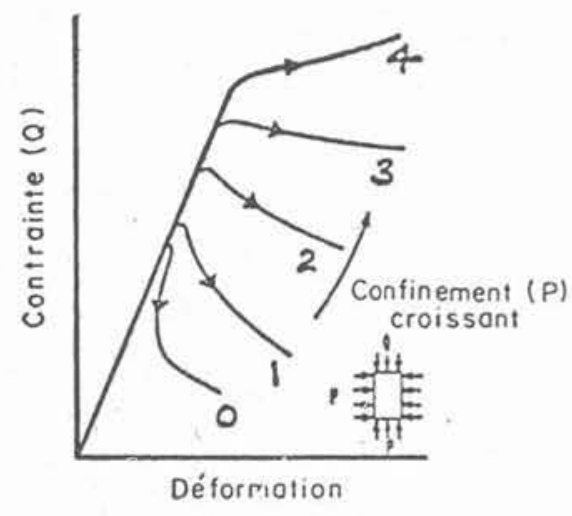

Fig. 6. - Comportement charge/déformation d'une couche de mine soumise à divers degrés de confinement.
Fig. 7. - Face avant du modèle analogique électrique utilisé pour le dimensionnement de l'extraction de la mine.

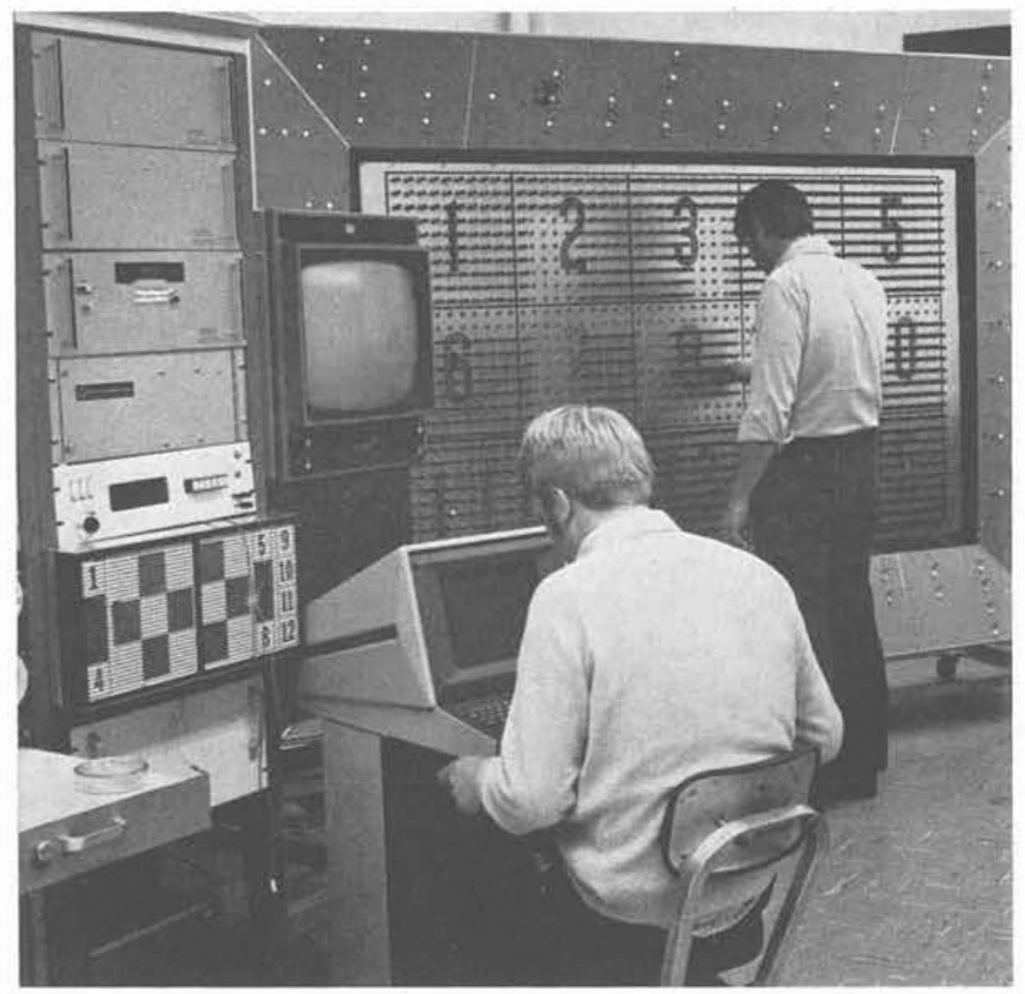

Un modèle analogique formé de résistances électriques a été construit à l'Université du Minnesota (semblable à celui décrit par Cook [9]), pour représenter la masse rocheuse élastique de part et d'autre du gisement. Lorsque ce dernier peut être supposé linéaire jusqu'à la rupture, des fiches, ayant une résistance électrique proportionnelle à la rigidité locale de la mine, sont insérées entre les noeuds du réseau et une plaque (d'aluminium) mise à la terre. Ces fiches simulent ainsi les éléments individuels constituant le gisement. Le courant qui passe coups de toits. Afin d'obtenir un tel accord, les résistances fixes sont remplacées par des sources à voltages variables couplées à chaque nœud. Ce voltage variable est contrôlé par une mini-calculatrice électronique de sorte que la relation courant/voltage (c'est-à-dire force/convergence). corresponde complètement au comportement force/convergence de chacun des éléments du gisement. Quand un modèle analogique est couplé de cette façon à un mini-ordinateur on a affaire à un système dit « hybride ». 


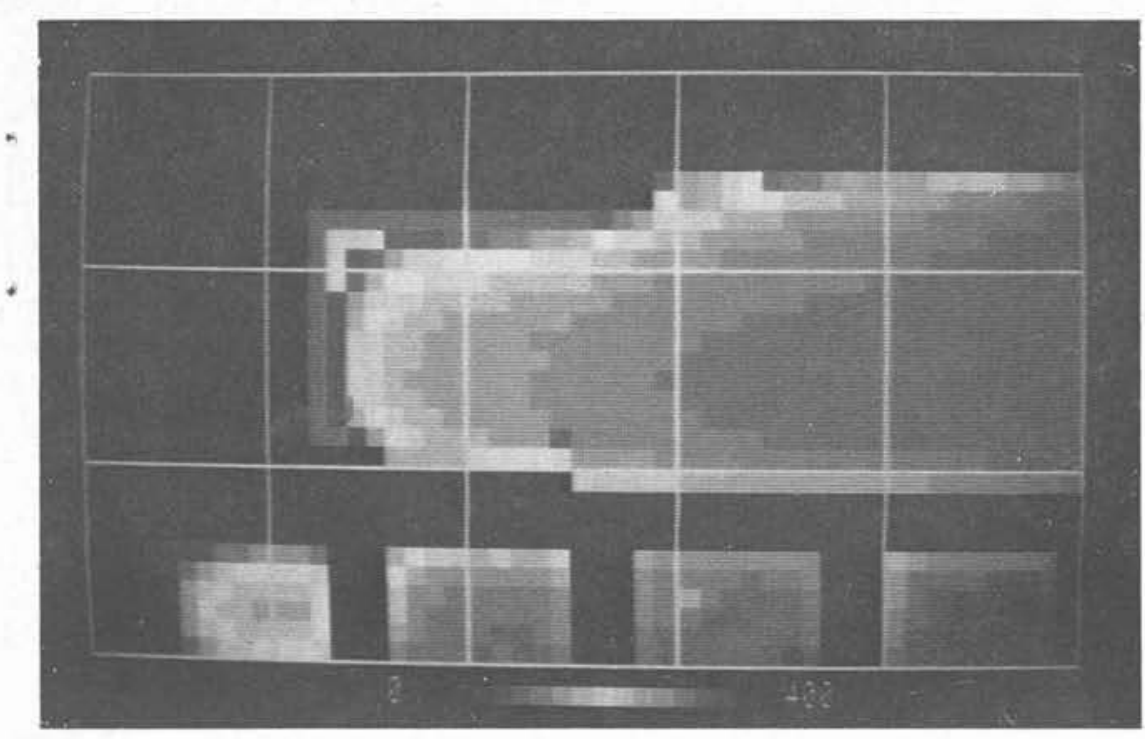

Fig. 8. - Résultats graphiques obtenus par « Ramtek »

La représentation graphique directe de la disposition de la mine sur le tableau des fiches est une caractéristique avantageuse du modèle analogique (fig. 7). A l'Université du Minnesota on y a ajouté un écran de télévision en couleur « Ramtek » (fig. 8) pouvant montrer le niveau de contrainte de chacun des nœuds, les différentes couleurs correspondant aux différents niveaux de contrainte. Les endroits critiques peuvent ainsi être rapidement détectés ainsi que la redistribution des contraintes résultant d'excavations supplémentaires. L'accès ainsi que les résultats graphiques sont un excellent moyen d'éducation et de communication.

La recherche actuelle est dirigée vers la détermination des caractéristiques appropriées pour les courbes (1) à (4) de la figure 6 dans le cas de gisements de charbon où des coups de toit sont fréquents. Des mineurs ainsi que des ingénieurs ayant une longue expérience pratique sont invités à simuler au moyen du modèle des séquences qui ont abouti à des coups de toit. Ils observent en même temps sur l'écran de télévision le développement des régions à fortes contraintes (et grands dégagements d'énergie) au fur et à mesure que l'extraction progresse. Les caractéristiques non linéaires utilisées pour le modèle sont modifiées jusqu'au moment où l'endroit du coup de toit observé coincide sur l'écran de télévision avec les régions à grands dégagements d'énergie. L'exercice est ensuite répété pour d'autres exemples. Cette recherche n'est pas encore terminée mais les premiers résultats indiquent que l'aspect graphique est extrêmement précieux car il permet une discussion fructueuse entre les chercheurs et les praticiens (ingénieurs et mineurs).

Nous espérons que cette méthode permettra la prévision du comportement du charbon dans la mine ainsi que le catalogage des expériences acquises.
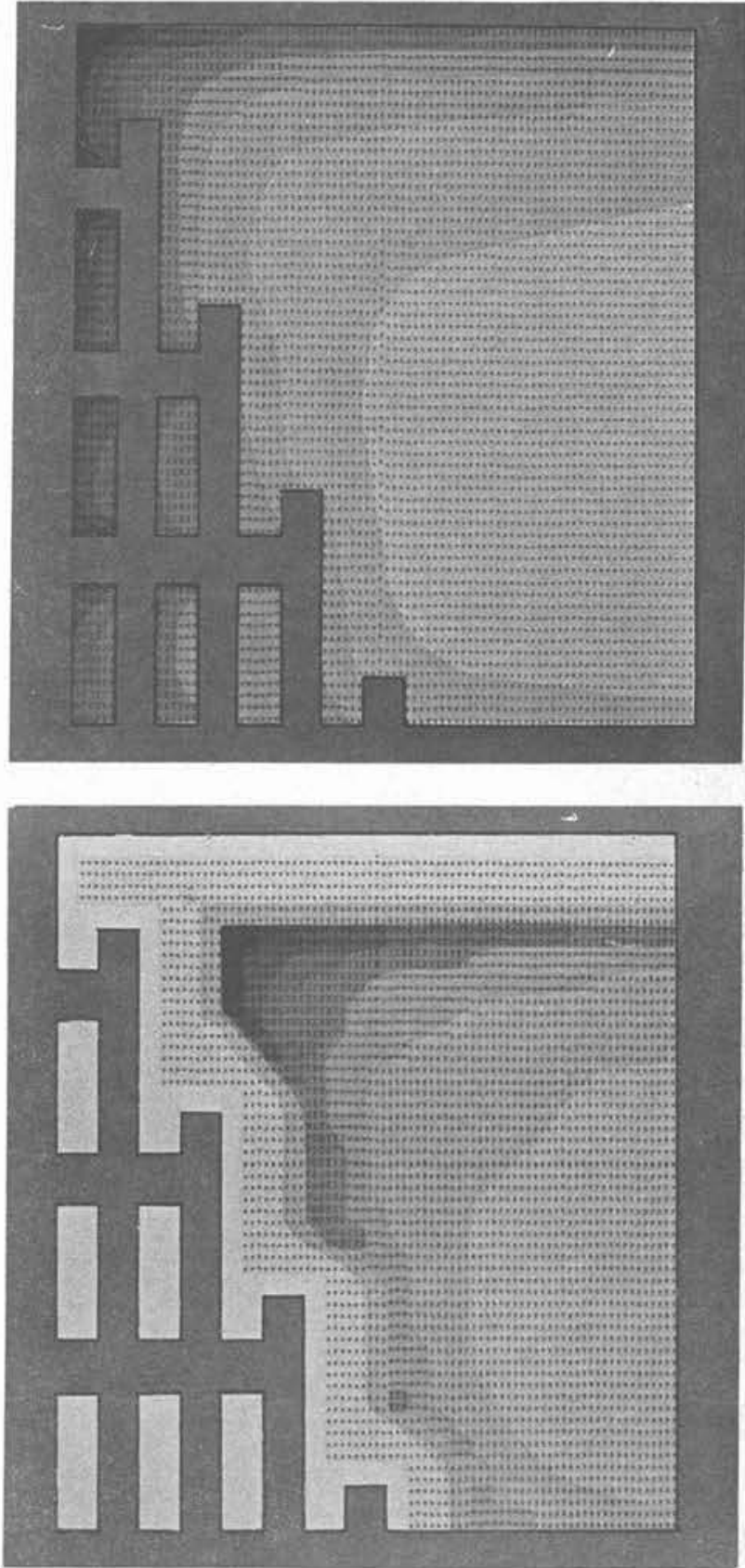

Fig. 9. - Distribution des contraintes verticales sur un film horizontal pour : (a) un comportement élastique ; (b) un comportement non élastique.

Des exemples sont donnés figure 9 dans le cas de comportement élastique et non élastique. Nous sommes convaincus que la méthode du modèle « hybride ", avec étalonnage à partir d'observations in situ, peut devenir un moyen pratique et efficace pour la définition des programmes d'extraction.

\section{ANALYSE DE LA STABILITE DES TUNNELS}

Le projet des tunnels offre un autre exemple d'utilisation de l'analyse énergétique. II est parfois suggéré que la forme optimale d'une excavation dans un champ de contraintes non hydrostatique $(P>Q)$ est une ellipse telle que le rapport $\frac{a}{b}$ soit égal à $\frac{\mathrm{P}}{\mathrm{Q}}$.
Il est vrai qu'un tunnel elliptique ayant son grand axe parallèle à la compression maximale comme indiqué figure $10(a)$ aura une distribution uniforme des contraintes $\sigma \theta$ à la paroi. Il n'est pas vrai cependant, comme c'est parfois avancé, que cette forme est la plus stable. En effet, bien que la contrainte tangentielle soit 


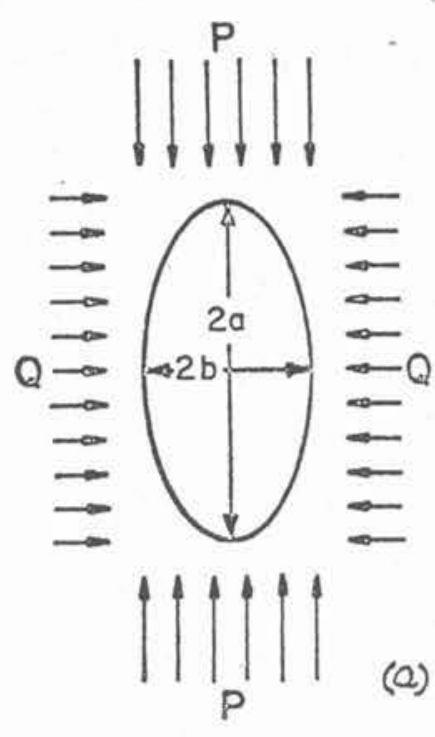

(a) Conception en fonction du concentration uniforme des contraintes

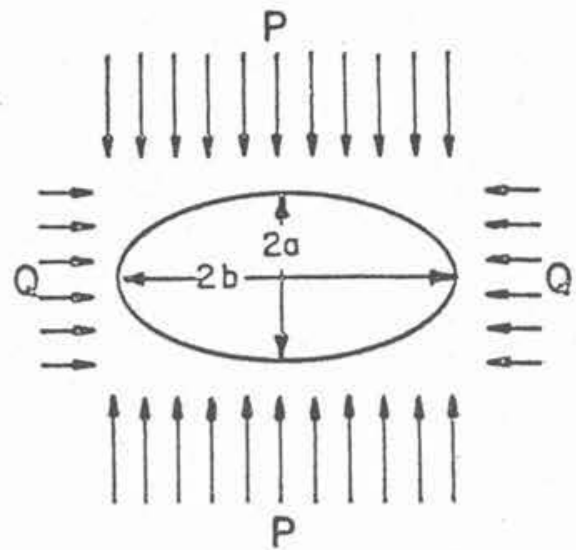

(b)

Fig. 10. - Orientations optimales d'un tumnel pour deux critères différents de conception.

constante sur les parois, le taux de décroissance de la contrainte tangentielle à l'intérieur du rocher massif est bien plus faible le long de l'axe horizontal (grand rayon de courbure) que le long de l'axe vertical (petit rayon de courbure). C'est-à-dire que l'énergie de déformation emmagasinée est plus grande dans la direction horizontale que dans la direction verticale. Donc, si le rocher est isotrope mais de résistance assez faible pour commencer à se rompre sous les concentrations de contraintes, l'extension de la rupture sera maximale dans la direction horizontale et minimale dans la direction verticale.

Pour présenter une stabilité maximale, le plus petit rayon de courbure doit correspondre en fait à la contrainte maximale et le plus grand rayon de courbure à la contrainte minimale; la forme la plus stable est alors l'ellipse indiquée figure $10(\mathrm{~b})$. c'est-à-dire ayant subi une rotation de $90^{\circ}$ par rapport à l'orientation habituellement admise.

Cet exemple est une illustration éloquente de la différence entre un dimensionnement basé sur un calcul de contrainte et un dimensionnement basé sur un calcul énergétique. Des exemples de rupture de tunnels elliptiques conçus en fonction des contraintes confirment que le tunnel se rompt de façon à se rapprocher de la forme stable, c'est-à-dire que la rupture au piédroit s'étend beaucoup plus que la rupture au toit.

En recommandant un critère de stabilité plutôt qu'un critère de contrainte dans les problèmes de résistance des roches, il faut préciser toutefois que l'analyse des contraintes ne doit pas être exclue. Il faut considérer la possibilité de rupture à proximité immédiate des excavations où a lieu une concentration de contraintes, l'analyse de la rupture étant poursuivie en utilisant une modélisation appropriée de la roche jusqu'à ce que le système se stabilise. Un exemple de cette approche est l'analyse par Daemen [10] de la « zone plastique » autour d'un tunnel non revêtu. Dans son analyse, utili- sant la méthode des éléments finis, Daemen a inclu l'effet de la dilatance du rocher en fonction de l'augmentation des contraintes. L'extension de la zone plastique autour d'un tunnel en fer à cheval et autour d'un tunnel cylindrique à proximité du front est indiquée figure 11 .

On voit dans les deux cas que la zone d'instabilité est minimale dans la zone du plus petit rayon de courbure (c'est-à-dire à la jonction front-piédroit) et maximale dans la zone du plus grand rayon de courbure (le front lui-même).

Daemen a également examiné l'effet stabilisateur de différents revêtements. Il observe que les cintres métalliques, bien qu'ayant une raideur intrinsèque très forte, ont souvent une raideur effective très faible à cause de l'influence prédominante du blocage (en bois) interposé entre le cintre et le rocher. Au contraire, le contact intime avec le rocher obtenu par le béton projeté est tel que la raideur d'une mince couche $(8 \mathrm{à} 10 \mathrm{~cm})$ de béton projeté peut être plus grande que celle d'un cintre métallique avec blocage en bois.

L'effet de la raideur sur la stabilisation d'un tunnel est indiqué figure 12 (b). Fairhurst et Singh [11] ont également considéré la stabilité d'un toit de mine stratifié, renforcé par des boulons scellés d'une façon similaire à celle utilisée pour un revêtement de tunnel, en fonction des forces de cisaillement actives (c'est-àdire dues au poids propre du rocher et aux contraintes latérales dans le toit) et des forces de cisaillement résistantes développées par des boulons scellés.

La force active est réduite par la déformation tandis que la force résistante augmente avec cette déformation. Une certaine déformation est nécessaire pour mobiliser la résistance du revêtement du tunnel (ou du boulon scellé) mais une trop grande déformation se se traduira par une rupture ou au mieux par un chargement du revêtement par le poids propre de la masse de rocher rompu. 
Fig. 11. - Extension de la zone fracturée autour:

a) d'un tunnel en fer à cheval b) d'un tunnel cylindrique dans un champ de contrainte hydrostatique.

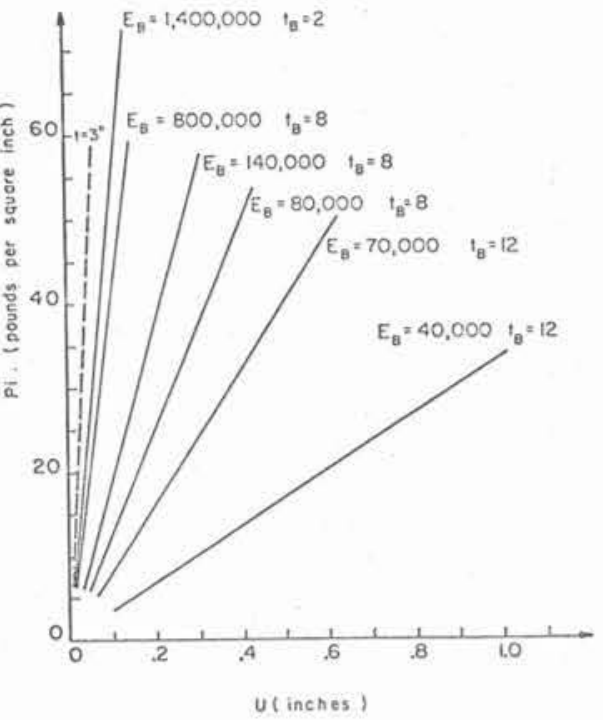

Fig. 12 a. - Caractéristiques de soutènement d'un cintre métallique bloqué au terrain, en fonction des paramètres de blocage.

Diamètre du tunnel $=16,7$ pieds. Espacement du blocage $=41,9$ pouces. Cintre de $6^{\prime \prime} \times 4^{\prime \prime}, 16 \mathrm{lbs} /$ pied, espacement $2^{\prime}$.

$\mathrm{E}_{\mathrm{B}}=$ module élastique du bloc en psi $-t_{B}=$ épaisseur du blocage en pouces. La ligne pointillée caractérise un revêtement en béton projeté de 3 pouces d'épaisseur, module de Young $\mathrm{E}=\mathbf{3 0 0 0 0 0 0} \mathrm{psi}$
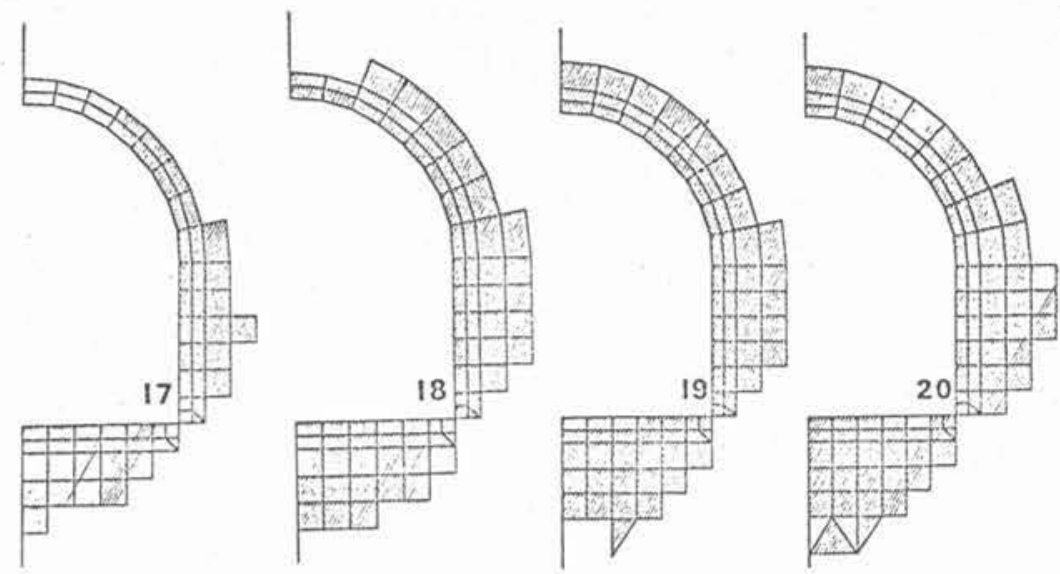

(a) "Rupture" progressive pendant les 4 dernières étapes d'un calcul de déchargement en 20 étapes.

Résistance à la compression uniaxiale $\sigma_{c}=250$ psi; champ de contrainte hydrostatique de c 1000 psi.

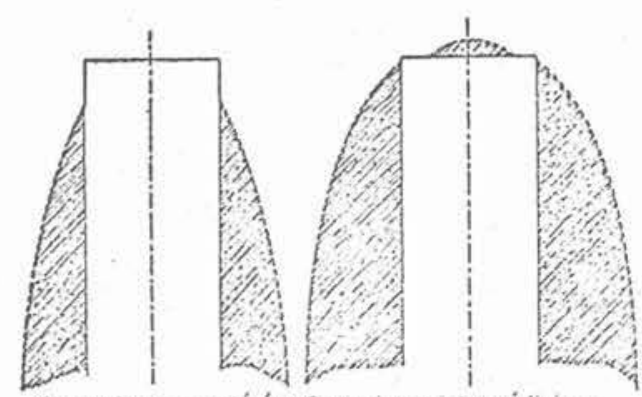

Controintes mocérées Contraintes intermédioires

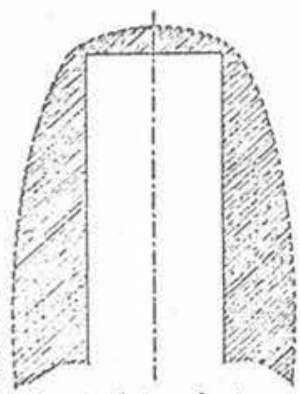

Contraintes fortes

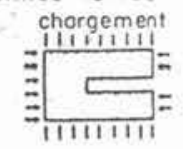

(b) Résumé schématique des zones de rupture au voisinage du front d'un tunnel pour un rapport croissant entre le champ de contrainte et la résistance de la roche.

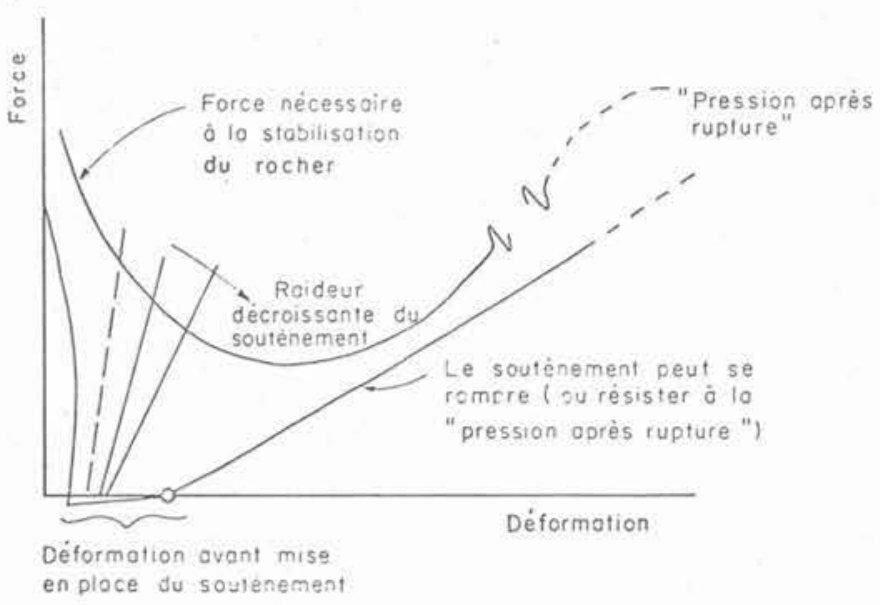

Fig. 12 b. - Effet de la rigidité du soutènement sur la stabilité d'une paroi de tunnel. 


\section{STABILITE D'UN MASSIF FORME D'UN ASSEMBLAGE DE BLOCS}

Jusqu'ici, nous avons supposé que la masse rocheuse se comportait comme un milieu continu. Il est bien connu que les discontinuités jouent un rôle primordial dans le comportement d'un massif mais l'analyse d'un milieu continu fournit des indications qui restent valables (par exemple effet d'un faible rayon de courbure, effet de la dilatation, effet de l'espacement des forages sur l'interaction entre fractures). On peut également estimer à quels déformations et mouvements conduit l'application sur la discontinuité du champ de contrainte calculé en milieu continu, ou estimer quel renforcement (ancrage) est nécessaire pour empêcher un glissement sur la discontinuité. Cependant une telle analyse devient rapidement trop complexe pour
1) Les lignes représentant la masse rocheuse, y compris les talus et les excavations, sont dessinées sur l'écran cathodique à l'aide d'un curseur.

2) L'ordinateur enregistre le système de lignes et identifie chaque bloc individuel.

3) Les blocs se déplacent suivant les lois physiques introduites dans le programme (loi reliant la force au déplacement; loi du mouvement). Des angles de frottement, des pressions d'eau, des renforcements divers peuvent être introduits.

Le programme a été décrit en détail par Cundall [14] ; le cycle de calcul est dans son principe donné ci-dessous :

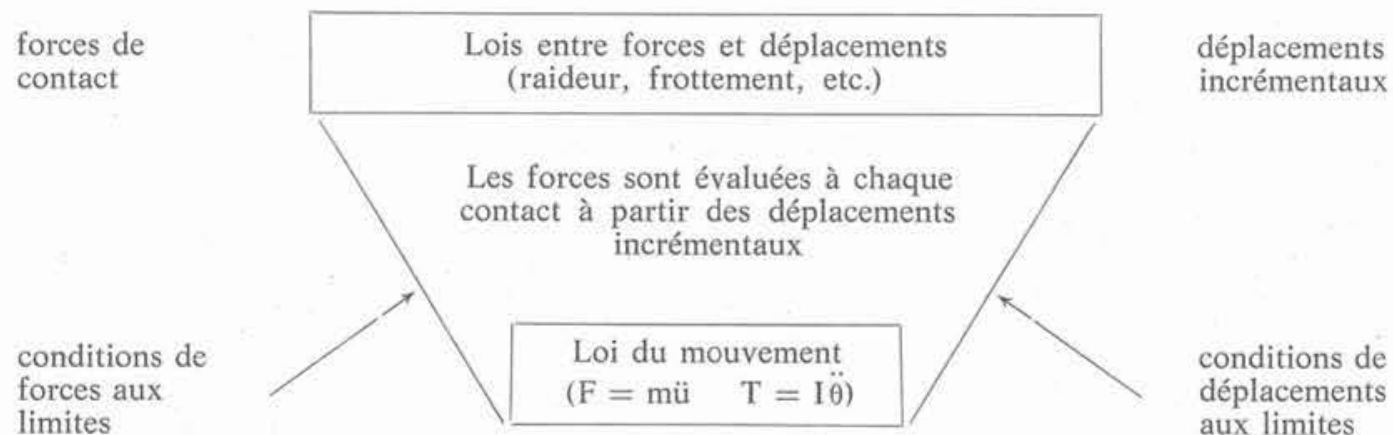

La résultante des forces sur chaque bloc provoque des accélérations qui sont intégrés deux fois pour calculer les déplacements sur un intervalle de temps $\Delta t$.

être applicable si le nombre de joints dépasse un ou deux.

Lorsque les discontinuités sont trop nombreuses pour ce type d'approche (Jaeger et Cook [12], p. 99), mais pas assez nombreuses pour que le massif puisse être considéré comme un milieu granulaire, il est important de tenir compte des discontinuités individuelles et de leurs propriétés.

L'établissement par Cundall [13] d'un modèle mathématique de blocs et son utilisation sur un ordinateur à sortie graphique (CIAG) est un progrès considérable. Bien qu'utilisé à l'heure actuelle principalement pour des analyses bidimensionnelles, il n'y a pas d'obstacle majeur à son utilisation pour des problèmes tridimensionnels. (L'Université du Minnesota est actuellement en train de modifier son ordinateur CIAG pour permettre le traitement de quelques problèmes tridimensionnels)

La méthode suppose que tous les déplacements ont lieu le long de joints ou discontinuités; cette hypothèse est réaliste pour un grand nombre de problèmes, particulièrement lorsque les joints sont altérés ou lorsque les contraintes normales sont faibles par rapport à la résistance à la compression de la matrice rocheuse. Les différences importantes avec les autres méthodes (équilibre limite et éléments finis) sont que le plan de rupture (ou les plans) n'est pas imposé mais se développe naturellement pendant le processus de calcul, et que les blocs peuvent subir de grands déplacements et de grandes rotations, se séparant même complètement les uns des autres si nécessaire. Le développement de l'instabilité apparaît graphiquement, de façon vivante, sur un écran cathodique. Trois étapes constituent l'analyse :
Le programme calcule l'équilibre des forces et des moments pour chaque bloc à chaque itération. Si un déséquilibre apparaît le bloc se déplace dans la direction de la force appliquée jusqu'à ce que l'équilibre soit rétabli, ou poursuit son mouvement si une condition de rupture a été atteinte.

Le programme est conçu pour une utilisation pratique. Les essais peuvent être faits sur l'écran exactement comme dans la réalité. Des blocs peuvent être enlevés, des chargements ajoutés, des blocs fixés puis relâchés et les forces mesurées.

L'angle de frottement d'un joint peut être modifié facilement et des pressions d'eau peuvent être introduites. Un problème complet peut être enregistré sur bande magnétique et utilisé plusieurs fois en faisant varier à chaque fois certains paramètres mais en conservant strictement la même géométrie initiale, ce qui n'est pas possible dans des modèles physiques.

Le plus grand attrait de ce système est peut-être la compréhension immédiate du problème par l'utilisateur et la stimulation intellectuelle procurée par la sortie graphique. II n'est pas nécessaire de comprendre le programme ou même d'essayer de lire les sorties d'ordinateur. L'utilisateur peut simplement vérifier le système en résolvant des problèmes qui lui sont familiers avant de commencer l'étude d'un problème nouveau. De cette façon, l'utilisateur se convainc lui-même de la validité du programme.

Comme pour le programme mis au point pour les mines, le système CIAG est un excellent véhicule de communication entre l'analyste théoricien et l'ingénieur de chantier praticien ou le géologue, qui peuvent souvent déterminer rapidement quel mode de rupture a réellement eu lieu dans des cas spécifiques. Ceci facilite 
le calcul des angles de frottement (et d'autres paramètres) les plus plausibles dans chaque cas.

La figure 13 montre comment le mode de rupture d'un talus (compliqué peut-être artificiellement pour illustrer la puissance du programme) change avec l'angle de frottement; il convient de noter que l'utilisation des méthodes de calcul par cercle de glissement aurait été tout à fait inappropriée ici, mais qu'avant la mise au point du programme de blocs aucune autre méthode n'existait.

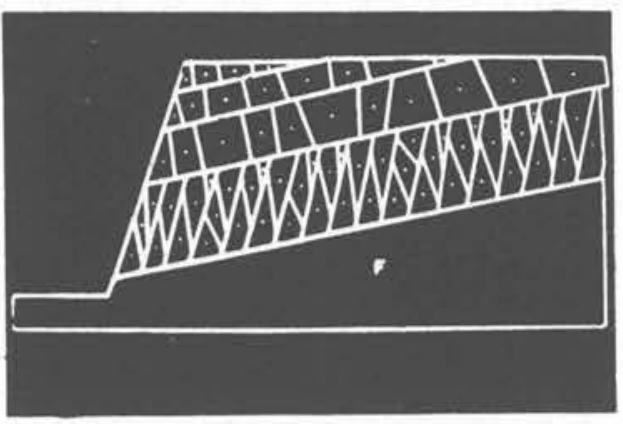

$\phi=40^{\circ}$ Stable

\section{Massif formé d'un assemblage de blocs}
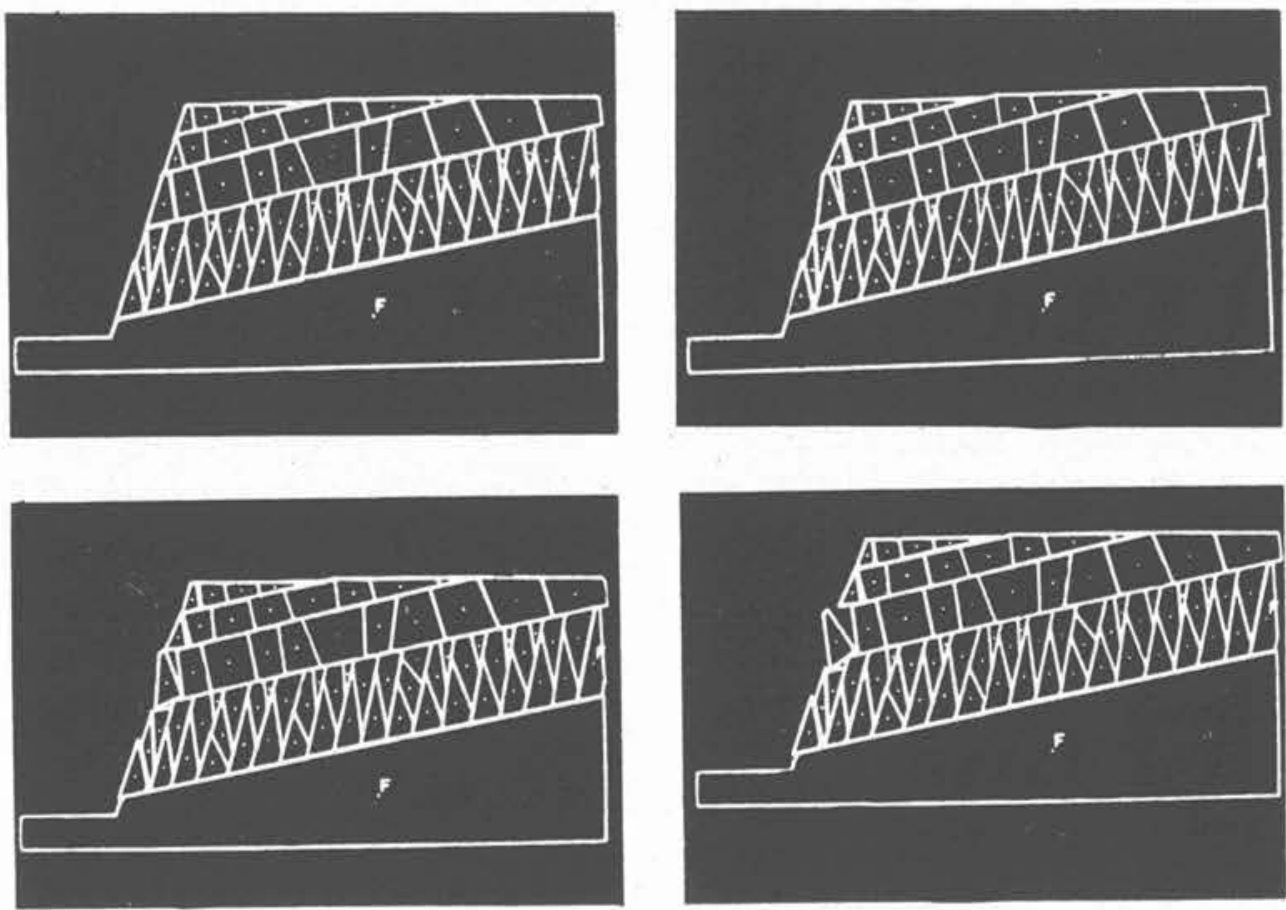

(a)

$$
\Phi=37^{\circ}
$$

Fig. 13. - Effet de l'angle de frottement des joints sur la stabilité d'un talus formé d'un assemblage de blocs.

\section{ANALYSE DES CONTRAINTES}

La longue série d'exemples dans lesquels l'analyse de la stabilité procure une meilleure compréhension physique de la situation ne doit pas cacher le fait qu'une analyse classique de contraintes apporte et continuera d'apporter des solutions intéressantes à bien des problèmes de mécanique des roches. D'une façon générale, les analyses de stabilité sont plus adaptées lorsqu'une certaine instabilité ou rupture est probable (par exemple au voisinage immédiat des excavations souterraines ou dans les essais de résistance) alors que l'analyse des contraintes est applicable à la détermination des déformations, à une certaine distance de l'excavation comme, par exemple, dans l'étude de la subsidence due aux mines ou aux redistributions de contraintes dans le massif rocheux. Des études sur l'utilisation simultanée des deux méthodes sont actuellement en cours. 

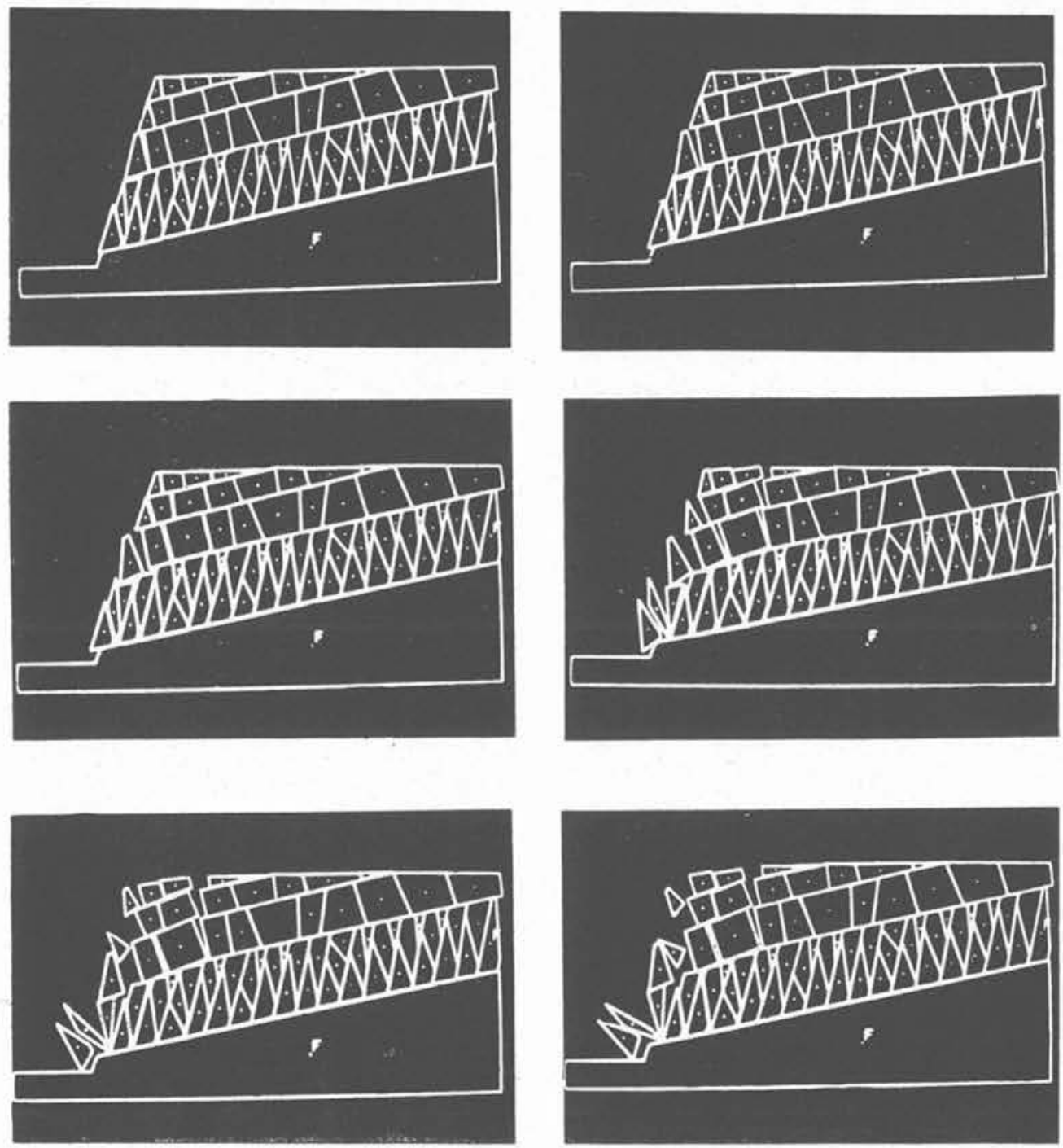

(b)

$\Phi=30^{\circ}$

Fig. 13 (suite)

\section{DIMENSIONNEMENT DES PILIERS DE SECURITE}

Un excellent exemple de la valeur pratique de l'analyse des contraintes en mécanique des roches est le problème du dimensionnement (diamètre) des piliers de sécurité, c'est-à-dire des piliers de minerai laissés en place à proximité d'une exploitation ou des piliers laissés sous une installation de surface de façon à éviter des basculements, distorsions, déformations ou subsidence excessifs qui pourraient gêner soit l'exploitation de la mine soit l'installation de surface. La figure 14 indique une dizaine de règles empiriques de dimensionnement des piliers d'après le Mining Engineers Handbook [15], [16].

Salamon [17] a utilisé l'analyse des contraintes pour déterminer, en fonction de la profondeur, la dimension du pilier produisant un effet constant en surface (tassement, basculement, etc.). Le résultat est indiqué figure 15 où est indiquée également la règle européenne souvent utilisée :

Rayon $\mathrm{R}$ du pilier $=0.7 \times$ profondeur $\mathrm{H}$ de l'exploitation.

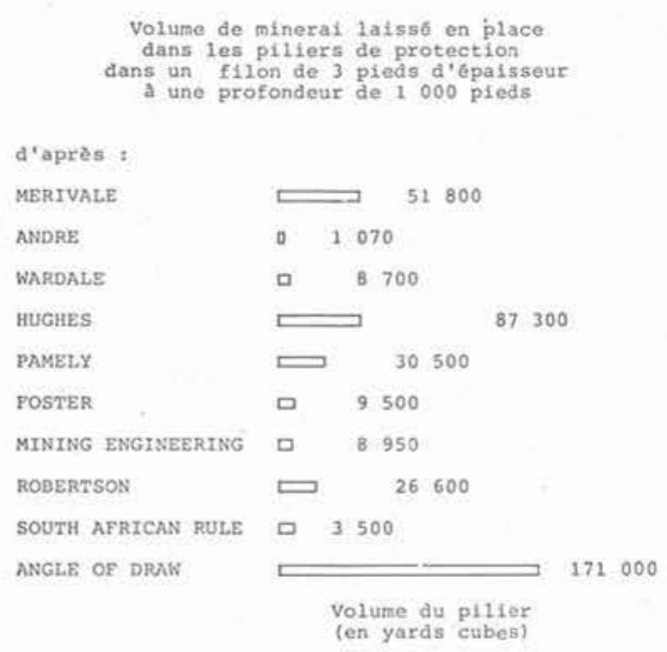

Fig. 14. - Variation des règles empiriques de dimensionnement des piliers de sécurité. 

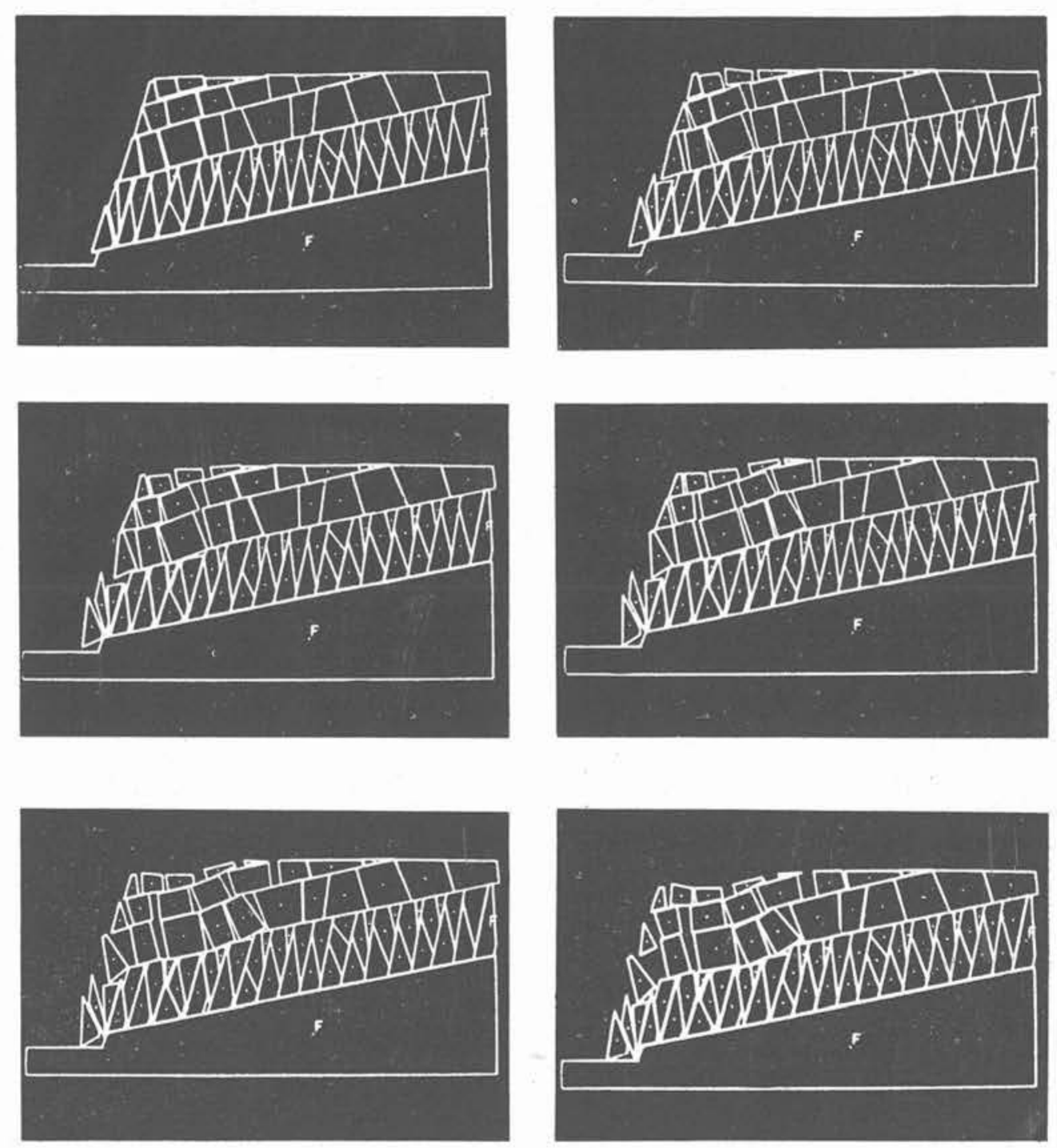

Fig. 13 (fin)

(c)

$$
\Phi=20^{\circ}
$$

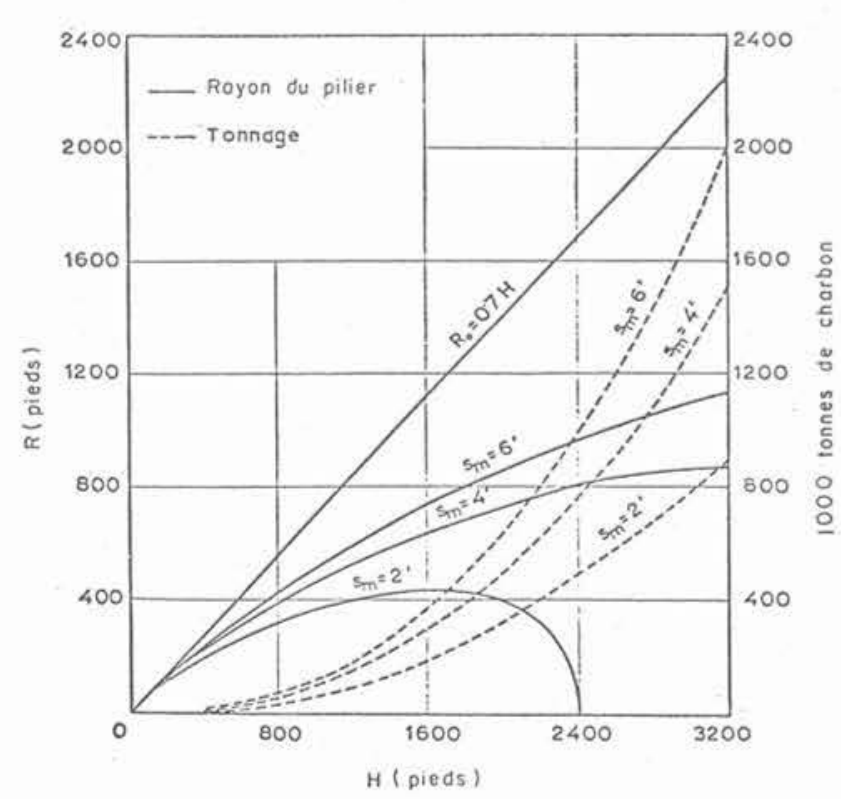

On peut voir que la théorie confirme la règle empirique pour des profondeurs faibles mais en diffère considérablement pour les grandes profondeurs. Il est intéressant de noter que la règle empirique d'Afrique du Sud, $\mathrm{R}=0.1 \mathrm{H}$, montre que des piliers plus petits que $\mathrm{R}=0.7 \mathrm{H}$ sont adéquats pour des mines profondes. L'utilisation de règles empiriques pour dimensionner les piliers de sécurité des mines profondes peut conduire à des conclusions fausses concernant la quantité de minerai exploitable. Dans des cas extrêmes, par exemple sous des zones où de nombreuses constructions doivent être protégées (et par conséquent où de nombreux piliers de sécurité sont à prévoir) ces règles empiriques peuvent conduire à de graves erreurs quant à la rentabilité économique de l'exploitation.

Des progrès ont été faits récemment dans les méthodes d'analyse des contraintes par le recours à la "Technique de déplacement des discontinuités " (Displacement Discontinuity Technique) [18], qui est une

Fig. 15. - Dimensionnement recommandé des piliers de sécurité à partir de l'analyse des contraintes (d'après Salamon), 
application du "principe de l'élément de paroi » (Face Element Principle) et de la " méthode de l'équation intégrale aux limites " (Boundary Integral Equation Method) [19]. Ces méthodes sont particulièrement bien adaptées aux problèmes traitant d'effets à distance de mouvements de discontinuités situées dans un milieu élastique. Ces méthodes sont également beaucoup mieux faites pour la programmation à l'ordinateur que l'application globale de la méthode des éléments finis à tout le massif rocheux.

\section{SIGNIFICATION DE L'ANALYSE}

Il convient de noter que dans cet article on a surtout utilisé les résultats de l'analyse pour éclairer qualitativement le comportement du massif rocheux et de la construction. Cette analyse est utile faute de valeurs numériques spécifiques pour les roches de chaque cas particulier.

Labasse [20] pose bien les problèmes généraux de mécanique des roches, commentant le problème du soutènement des tunnels :

« Avant d'attaquer ce problème, il faut le poser de la façon dont il se pose dans la pratique. D'abord, il est nécessaire de ne pas avoir plus d'une ou deux dimensions d'arc ou de voussoir si on veut éviter une confusion à la mise en place. Une telle standardisation rend inutiles les calculs précis du soutènement pour chaque section du tunnel. De plus, on peut toujours faire varier l'espacement des éléments de soutènement comme on le désire. Enfin, la nécessité de mettre en place le soutènement immédiatement après l'excavation laisse un temps insuffisant pour le calcul et la fabrication du soutènement idéal pour une section particulière du tunnel. Il serait nécessaire, si l'on voulait arriver à un dimensionnement exact, d'étudier chaque section séparément puisque chaque section diffère de la précédente par la nature des terrains rencontrés, leur pendage et leur disposition. Il faudrait faire des prélèvements dans chaque couche pour déterminer ses propriétés et l'influence de ces propriétés sur celles de la couche voisine. Tout ceci conduirait à une série d'essais et d'équations dont la solution, en admettant qu'il soit possible de trouver une solution, ferait perdre un temps précieux pendant lequel le tunnel s'effondrerait certainement. "

Il n'est jamais possible de concevoir un ouvrage sur la base de valeurs numériques précises des propriétés mécaniques des roches. Le projeteur doit reconnaître ce fait. La dispersion est inévitable et doit être prise en compte. Mais ceci ne veut nullement dire que l'analyse théorique n'est pas importante. Il est très utile par exemple de connaitre le poids des variables en cause - il arrive que certaines variables peuvent changer considérablement en ayant peu ou pas d'effet sur la stabilité générale de la structure ou sur la conception définitive. D'autres variables peuvent être plus critiques. Hoek et Londe [21] notent que l'analyse de la sensibilité d'un projet aux paramètres en cause a beaucoup plus de signification que le calcul de coefficients de sécurité "spécifiques".

La mise en place de soutènements dans la nouvelle méthode autrichienne est un bon exemple de conception intelligente en mécanique des roches. Des analyses théoriques montrent que si les parois du tunnel pouvaient se déformer sans se rompre elles tendraient à devenir autostables et se stabiliseraient. Un soutènement artificiel est habituellement nécessaire pour maintenir l'intégrité de la paroi rocheuse mais le type et la quantité de soutènement ne peut pas être calculé pour chaque section dans un terrain variable.

Pour cette raison, les déformations radiales sont mesurées après le traitement initial (ancrage, cintres légers en acier, béton projeté ou une quelconque combinaison de ces méthodes) des parois que l'on vient d'excaver. $\mathrm{Si}$ les déformations tendent à s'accélérer, la situation est instable et un soutènement supplémentaire est mis en place jusqu'à ce que la déformation se stabilise à une valeur finale acceptable. Le soutènement d'un tunnel est conçu en se servant de sa souplesse d'emploi de façon à faire face à un besoin variable imposé par la géologie. C'est le contraire d'une conception où tous les soutènements sont « conçus » et fabriqués avant que le rocher soit excavé et installés uniformément avec peu de mesures vérifiant le bien-fondé du système mis en place.

\section{CONCLUSION}

Il est clair que l'analyse théorique a un rôle utile à jouer dans la mise en œuvre pratique de la mécanique des roches. L'utilisation la plus féconde d'une telle analyse est dans le développement d'une compréhension meilleure de la base mécanique et physique des problèmes ainsi que des conséquences éventuelles de variations par rapport à une condition initiale supposée. Une analyse de stabilité est nécessaire lorsque la masse rocheuse peut supporter des contraintes importantes, par exemple au voisinage immédiat des parois d'excavation. L'analyse des contraintes est plus appropriée pour les cas où les changements de contraintes sont relativement faibles ou lorsque la charge appliquée est telle que l'instabilité est inévitable dès que la résistance est dépassée (par exemple, fondations de barrage ou stabilité des talus sous l'effet de leur poids propre).

Eclairé par ces analyses, le projeteur sera mieux équipé pour répondre à des conditions variables. Les bons projets en mécanique des roches sont ceux qui permettent de faire face rapidement à de telles variations.
Une meilleure connaissance des propriétés mécaniques des massifs rocheux est nécessaire pour améliorer les projets en mécanique des roches. Un moyen économique d'obtenir des données est de comparer les comportements des ouvrages avec les prédictions des modèles théoriques utilisés pour le projet. L'utilisation de graphes « interactifs " (méthode conversationnelle sur écran cathodique) peut être un moyen très efficace de stimuler un échange créatif entre le calculateur, le projeteur et l'homme de chantier, et par conséquent faciliter le nécessaire dialogue entre la théorie et la pratique et, finalement, conduire à une meilleure mécanique des roches.

En résumé, les chercheurs en mécanique des roches utilisent la mécanique classique théorique mais la nature des matériaux géologiques nous impose une philosophie quelque peu différente dans l'application pratique de la mécanique - approche dans laquelle les variations par rapport aux conditions supposées d'analyse jouent un grand rôle dans la conception et dans laquelle un dialogue entre le chercheur et l'utilisateur est essentiel. 


\section{REMERCIEMENTS}

Les propos de cet article proviennent de nombreuses sources et résultent d'influences multiples au cours de longues années.

Je suis reconnaissant à tous et particulièrement aux étudiants diplômés qui ont, par un travail laborieux, établi les résultats concrets d'où se dégageront des lois générales.
Je remercie également les différentes sources d'aide financière dispensée ces dernières années : The Petroleum Research Fund, American Petroleum Institute, Advanced Research Project Agency National Science Foundation, RANN Excavation Technology Program, Department of Transportation, US Bureau of Mines, Cold Spring Granite Company et d'autres.

\section{ANNEXE}

\section{THEORIE DE LA RUPTURE DE GRIFFITH}

Bien qu'habituellement citée en relation avec les aspects micro-structuraux de la rupture des matériaux fragiles, les principes de base de la Théorie de Griffith sont applicables aussi à la rupture à grande échelle et il est instructif de les rappeler. Griffith indique que la rupture d'un solide chargé implique deux conditions nécessaires :

\section{1) Condition de contrainte}

En un point quelconque du solide, la contrainte locale doit être suffisamment grande pour vaincre la force de cohésion de la roche (c'est-à-dire les forces d'attraction entre les particules).

\section{2) Une condition d'énergie}

Une énergie suffisante doit être libérée pour alimenter continuellement l'énergie de surface des deux surfaces nouvelles formées par la fissure qui se propage.

Griffith suggère que c'est aux extrémités des microfissures préexistantes dans le solide que la condition de contrainte peut être satisfaite. Dans une roche, il y a toujours suffisamment d'hétérogénéité, de fissures, et de frontières entre grains pour provoquer la concentration de contraintes nécessaire pour satisfaire la première condition; on peut, par conséquent, concentrer son attention sur la seconde condition.

Comme on l'a dit plus haut, la condition d'énergie est simplement une application du théorème de l'énergie potentielle minimale. (" L'état d'équilibre stable d'un système est celui pour lequel l'énergie potentielle du système est minimale »).

Griffith a appliqué ce théorème de façon spécifique à la rupture en ajoutant l'énoncé suivant : " La position d'équilibre (si l'équilibre est possible), doit être une position dans laquelle la rupture du solide a eu lieu, si le système peut passer d'une condition intacte à une condition de rupture par un processus comportant une diminution continuelle de l'énergie potentielle " [1].

Il a appliqué le théorème au cas d'une plaque d'épaisseur unitaire, de module d'élasticité E, tendue de façon uniaxiale (fig. A 1) en calculant la variation d'énergie potentielle du système due à l'introduction d'une fine fissure de longueur $2 c$. Il a démontré que la variation d'énergie potentielle est égale à $\Delta \mathrm{P}$, où

$$
\Delta \mathrm{P}=4 c \gamma-\frac{\pi c^{2} \sigma^{2}}{\mathrm{E}}
$$

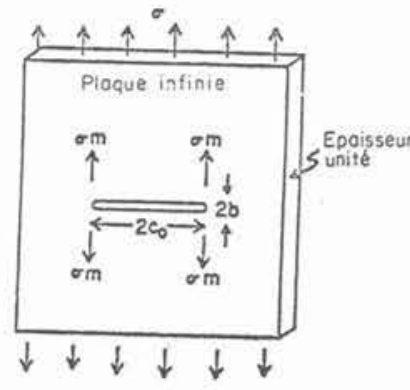

(a)

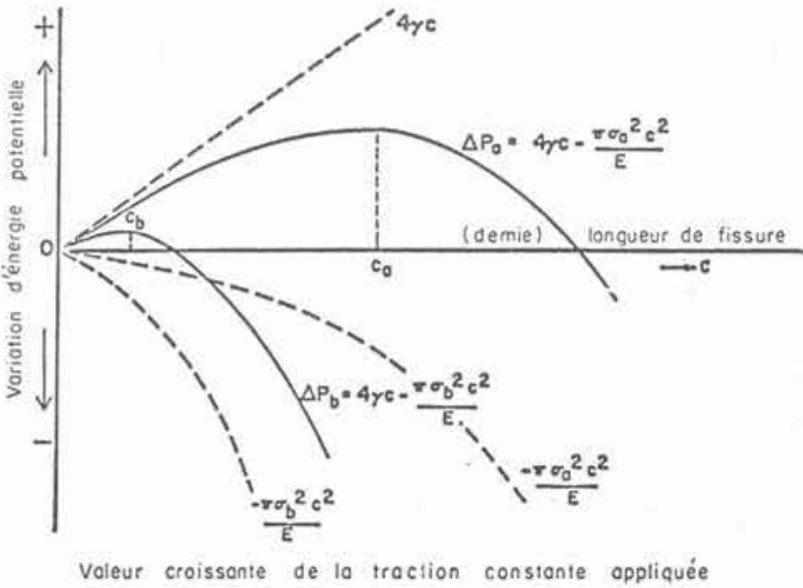

(b) 
où :

$4 c$ est l'augmentation de surface due aux faces supérieure et inférieure de la fissure de longueur $2 c$.

$\gamma$ est l'énergie de surface spécifique de la surface de la fissure.

$4 c \gamma$ est l'augmentation d'énergie de surface due à l'introduction de la fissure.

$-\frac{\pi c^{2} \sigma^{2}}{E}$ est la variation (diminution) d'énergie due à la variation $(a)$ de l'énergie potentielle des forces appliquées et $(b)$ à l'énergie de déformation de la plaque chargée.

L'équation A (1) est donnée sous forme graphique figure A 1 (b).

Pour un niveau de contrainte donné $\sigma=\sigma_{a}$, l'introduction d'une fissure très légèrement plus longue que $2 c=2 c_{e}$, produira, d'après Griffith, la rupture puisque l'énergie potentielle du système va décroître continuellement pour $c>c$. En dérivant l'équation $\mathrm{A}$ (1) par rapport à la longueur de la fissure $c$ et égalant la dérivée à zéro on trouve la relation entre la contrainte critique $(\sigma)$ et la longueur de fissure $(c)$ :

$$
\begin{aligned}
\sigma & =\sqrt{\frac{E_{\gamma}}{\pi c}} \\
\sigma^{2} c & =\frac{E_{\gamma}}{\pi}=\text { constante }
\end{aligned}
$$

Il faut noter que la longueur de la fissure apparaît dans l'équation A (2) parce que la libération d'énergie s'accumule dans le volume de la plaque (d'épaisseur unité) alors que la demande d'énergie est proportionnelle à la surface de la fissure.

Berry [22] a établi les courbes contraintes uniaxiales/déformations pour le système de plaque de Griffith. Il a défini un module effectif $E^{\prime}$ pour la plaque fissurée, à laquelle il attribue une surface $\mathrm{A}$, de la façon suivante :

- Energie de déformation de la plaque chargée non fissurée : $\frac{\mathrm{A} \sigma^{2}}{2 \mathrm{E}}$

- Augmentation d'énergie de déformation due à la fissure : $\frac{c^{2} \sigma^{2}}{\mathrm{E}}$

(Sneddon [23]).

- Energie de déformation de la plaque fissurée $\frac{\mathrm{A} \sigma^{2}}{2 \mathrm{E}^{\prime}}=\frac{\mathrm{A} \sigma^{2}}{2 \mathrm{E}}+\frac{c^{2} \sigma^{2}}{\mathrm{E}}$

d'où $\mathrm{E}^{\prime}=\frac{\mathrm{E}}{1+\frac{2 \pi c^{2}}{\mathrm{~A}}}$

En combinant l'équation $\mathrm{A}$ (1) et l'équation $\mathrm{A}$ (3) avec la relation élastique $\sigma=E^{\prime} \varepsilon$ Berry a obtenu la condition suivante de rupture en traction :

$$
\varepsilon_{\mathrm{g}}=\frac{\varepsilon_{\mathrm{g}}}{\mathrm{E}}+\frac{8 \mathrm{E} \gamma^{2}}{\mathrm{~A} \pi \delta_{g}}
$$

Cette condition est représentée graphiquement figure A 2 .

On peut voir que la courbe de rupture est asymptotique à une droite de pente égale au module intrinsèque du matériau (c'est-à-dire sans fissure). Considérons une plaque contenant une fissure telle que le module soit défini par la pente O 1. A un niveau de contrainte $\sigma$, le critère de Griffith est satisfait et la fissure va s'étendre. Si le niveau de contrainte est réduit par l'extension de la fissure de façon à suivre exactement la courbe $(1,2,3)$ le critère de l'équation $\mathrm{A}(2)$ est

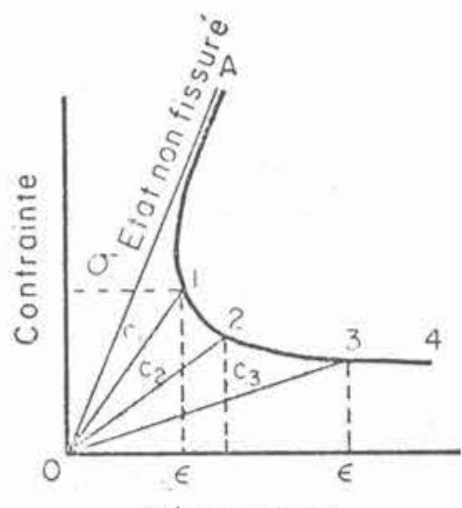

Déformation

Fig. A 2. - Courbe du critère de Griffith pour une rupture en traction.

continuellement juste satisfait et la fissure se propage de façon quasi-statique sans accélération, c'est-à-dire en fournissant exactement l'énergie de surface nécessaire pour s'étendre comme représenté par exemple par la surface hachurée $0,1,2,3$, de la figure A 2 dans laquelle la fissure s'étend d'une longueur $c_{1}$ à une longueur $c_{3}$.

Il faut noter que la surface $\mathrm{O} z \varepsilon_{3}$ représente l'énergie élastique de déformation de la plaque à un niveau de contrainte 3 et pour une longueur de fissure $c 3$.

$\mathrm{Si}$ cependant la contrainte appliquée $\sigma$ diminue suivant un autre chemin que le chemin de rupture, la condition d'équilibre d'énergie de l'équation A 2 ne sera pas satisfaite.

Considérons par exemple la figure A 3 . La contrainte appliquée est augmentée jusqu'à une valeur $\sigma_{1}$ et l'extension de la fissure commence. Mais maintenant la contrainte diminue en suivant le chemin 1-S déterminé par la raideur du ressort.

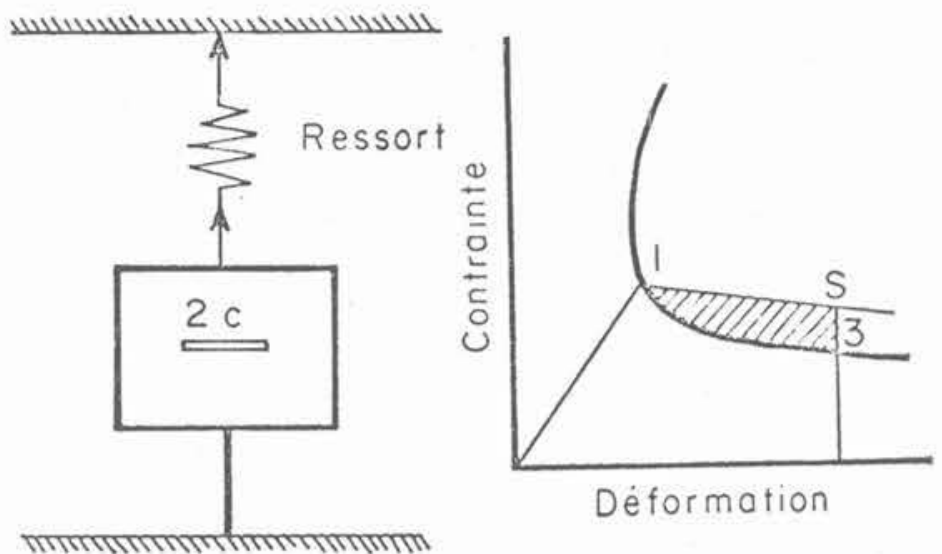

Fig. A 3. - Extension instable d'une fissure.

Dans ces conditions l'énergie fournie par le ressort dépasse l'énergie nécessaire pour causer une propagation statique de la fissure d'une valeur proportionnelle à l'aire hachurée $1 \mathrm{~S} 3$ dans la figure A 3 . L'excédent d'énergie accélère la propagation de la fissure et une rupture instable s'ensuit. Naturellement, si la rigidité du ressort est telle que le chemin $1 \mathrm{~S}$ tombe en dessous du lieu de rupture de Griffith, la fissure ne s'étendra pas, à moins que la contrainte appliquée au ressort soit continuellement augmentée pour suivre ce chemin. Donc une rupture instable a lieu lorsque l'énergie fournie excède l'énergie dissipée (l'énergie de surface dans le modèle de Griffith). 


\section{APPLICATION DES ANALYSES DE GRIFFITH ET DE COOK A LA FRACTURATION DES ROCHES}

Les analyses de Griffith et de Cook offrent certainement un schéma trop simplifié et idéalisé de la rupture des roches.

Il est bien connu par exemple que l'énergie nécessaire pour séparer les faces des fissures dans une roche est beaucoup plus importante que l'énergie de surface. Une énergie considérable est dissipée dans la micro-fracturation, la rotation des grains, etc., à proximité de la direction principale de fracture : l'énergie également ne croît pas de façon uniforme avec la longueur de la fissure à cause de l'hétérogénéité de la roche.

En compression, l'extension initiale de la fracture tend à avoir lieu parallèlement à la direction de la plus grande contrainte principale et non dans la direction du cisaillement.
Cependant, la rupture finale a lieu habituellement en cisaillement. Ces critiques peuvent être généralisées en appelant « $\delta$ » le « travail de rupture », c'est-à-dire la demande d'énergie " totale " pour un accroissement unitaire de longueur de la fissure en extension; et de façon similaire en appelant $\gamma_{s}$ la demande d'énergie " totale " pour la propagation du cisaillement.

La conclusion générale qui reste qualitativement valable est que la stabilité d'une fracture peut être examinée en comparant l'énergie fournie par l'extension de la fracture à l'énergie dissipée pendant cette même extension. Ce principe général peut être appliqué de façon pratique en mécanique des roches, comme on l'a montré dans le corps de l'article.

\section{RUPTURE EN COMPRESSION}

Cook [24] a étendu l'analyse de Griffith à la propagation d'une fissure inclinée sur la direction d'une compression uniaxiale et montre que le critère de rupture fragile en cisaillement est le suivant :

$$
\tau-\mu \sigma_{n}=\sqrt{\frac{\mathrm{E} \gamma}{\pi c}} s
$$

où

$\sigma_{n}, \tau$ sont les contraintes normales et de cisaillement sur la fissure inclinée d'un angle $\alpha$ sur la direction de compression.

$\gamma_{s} \quad$ est l'énergie de surface nécessaire à la propagation de la fissure.

$\mu \quad$ est le coefficient de frottement le long de la fissure.

Il est intéressant de noter que l'équation A (5) est essentiellement le critère de Mohr-Coulomb. Cook a également étendu son analyse à la rupture en compression triaxiale.

\section{REFERENCES}

[1] GRIFFITH (A.A.). - Phenomenon of Rupture and Flow of Solids, Trans. Roy. Soc. (London) A 221, pp. 163-198, 1921.

[2] GRIFFITH (A.A.). - The Theory of Rupture, Proc. Ist. Int. Cong. Appl. Mech, Delf, 1924, pp. 55-63.

[3] KNOTT (J.-F.). - Fundamentals of Fracture Mechanics, (New York), 1973, 273 p.

[4] HARDY (M.P.) and FAIRHURST (C). - Analysis of Fracture in Rock and Rock Masses, American Society of Mechanical Engineers, Albuquerque Division, Spring Meeting, February 1974, pp. 73-80.

[5] OUCHTERLONY (F.). - Fracture Mechanics Applied to Rock Blasting, Proc. 3rd ISRM Congress, Denver, 1974, Volume II-B, pp. 1377. 1383.

[6] BLIGH (T.P.). - Principles of Breaking Rock Using high Pressure Gases, Proc. 3rd ISRM Congress, Denver, 1974, Volume II-B, pp. 1421 . 1427.

[7] CORNET (F.). - Hard Rock Blasting Developments and Possibilities, Atlas Copco's Bench Drilling Days, June, 1975, Stockholm, 11 p.

[8] IRWIN (G.R.). - Fracture Handbuch der Physik, Vol. VI, Springer (Vienna).
[9] COOK (N.G.W.). - « The Design of Underground Excavations », Proc. 8th Symposium on Rock Mechanics, AIME, 1967, pp. 167-193.

[10] DAEMEN (J.J.K.). - Tunnel Support Loading Caused by Rock Failure, U.S. Corps of Engineers Technical Report MRD-3-75, May 1975.

[11] FAIRHURST (C.) and SINGH (B.). - Roofbolting in Horizontally Laminated Rock, Engineering and Mining Journal, February, 1974, pp. 80-90.

[12] JEAGER (J.C.) and COOK (N.G.W.), - Fundamentals of Rock Mechanics, London, Methuen, $1969,513 \mathrm{p}$.

[13] CUNDALL (P.), VOEGELE (M.D.) and FAIRHUST (C.). - Computerized Design of Rock Slopes Using Interactive Graphics for the Input and Output of Geometrical Data, Proc. 16th Symp. on Rock Mechanics, Minneapolis, 1975, pp. $1-10$.

[14] CUNDALL (P.A.). - Rational Design of Tunnel Supports: A. Computer Model for Rock Mass Behavior Using Interactive Graphics for the Input and Output of Geometrical Data, U.S. Army Corps of Engineers Technical Report MRD2-74, 1974.

[15] PEELE (R.). - Mining Engineers Handbook, Wiley (New York) 1941, deux volumes. 
[16] DAEMEN (J.J.K.). - The Effect of Protective Pillars on the Deformation of Mine Shafts, Rock Mechanics, Journal of the International Society for Rock Mechanics, Volume 4, $\mathrm{n}^{\circ}$ 2, October 1974, pp. 89-115.

[17] SALAMON (M.D.G.). - An Elastic Analysis of Displacements and Stresses Induced by Mining of Seam or Reef Deposits, Part III, Journal South African Institute of Mining and Metallurgy, Vol. 64, pp. $468-500$.

[18] CROUCH (S.L.). - Solution of Plane Elasticity Problems by the Displacement Discontinuity Method, Parts I and II, International Journal for Numerical Methods in Engineering, Vol. 10, 1976, pp. 301-343.

[19] RIZZO (F.J.). - An Integral Equation Approach to Boundary Valve Problems of Classical Elastotatics, Quart. of App. Math 25, 83-95 (1967).

[20] LABASSE (H.). - Les Pressions de Terrains dans les Mines de Houilles, III - Les Pressions de Terrains autour des Travers - Bancs Horizontaux,
Revue Universelle des Mines, Junuary, 1950. pp. 3-22.

[21] HOEK (E.) and LONDE (P.). - Surface Workings in Rock, Proc. 3rd ISRM Congress, Denver, 1974, Vol. I-A, pp. 613-654.

[22] BERRY (J.-P.). - Some Kinetic Considerations of the Griffith Criterion for Fracture. I. Equations of Motions at Constant Force, Jour. Mech. Phys. Solids, Vol. 8, pp. 194-206.

[23] SNEDDON (J.N.). - The Distribution of Stress in the Neighbourhood of a Crack in an Elastic Solid, Proc. Roy. Soc. London Series A, Vol. 187, pp. $229-260$.

[24] COOK (N.G.W.). - The failure of Rock, Int. Journ. Rock Mech. Min. Sci., Vol 2, pp. 389-403.

Voir aussi FAIRHURST (C.) and COOK (N.G.W.). The Phenomenon of Rock Splitting Parallel to the Direction of Maximum Compression in the Neighbourhood of a Surface, Proc. Ist. Cong. Rock Mechanics, Lisbon, 1966, pp. 687-692. 